\title{
Effets d'un obstacle parallélépipédique sur la propagation de la houle
}

\section{The effects of a rectangular obstacle on wave propagation}

\author{
PAR K. TAKANO \\ ATTACHÉ DE RECHERCHES DU C.N.R.S. \\ LARORATOIRES DE MÉCANIQUE DES FLUJDES DE L'E,N.S.E.H.R. DE GRFNOBLE
}

\begin{abstract}
Le présent mémoire est consacré à l'étude théorique, dans le cadre de l'approximation linéaire, de deux phénomènes, voisins au point de vue analytique, mais distincts au point de vue physique. Considérons un canal $\dot{a}$ houle ouvert, $\dot{a}$ fond horizontal, $\dot{a}$ section rectangulaire. Un obstacle parallélépipédique $\mathrm{S}$ de largeur égale à celle du canal, à bases horizontales, peut ètre utilisé de deux manières pour troubler la propagation de la houle plane.

$1^{\circ} \mathrm{S}$ est partiellement immergé dans le liquide. La houle incidente subit une réflexion partielle sur la face amont de $\mathrm{S}$, traverse en charge l'espace compris entre le fond du canal et la base intérieure de $\mathrm{S}$, puis réapparait à l'aval de $\mathrm{S}$ sons forme de houle transmise.

$2^{\circ} \mathrm{S}$ est complètement immergé : la houle incidente passe par dessus le seuil ainsi constitué, engendrant au-dessus de $S$ et $\dot{a}$ l'awal, une agitation périodique de liquide qui peut etre fort complexe.

Notre truail se trouve ainsi naturellement divisé en deux parties. Ponr la commodité du lecteur, nous les abons rédigées d'une manière indépendante l'ane de l'antre.
\end{abstract}

\begin{abstract}
This article is devoted to a theoretical treatment by means of linear approximations of two analytically similar but physically distinct phenomena. Consider an open wave flume with a horizontal bottom. A rectangular obstacle $\mathrm{S}$, the top and bottom of which are parallel, and whose width is the same as that of the flume, can disturb two-dimensional wave propagation in two ways :

$1^{\circ} \mathrm{S}$ is partially immersed in the liquid. The incident waves are partially reflected by the upstream face of $\mathrm{S}$, pass between the lower face of $\mathrm{S}$ and the bottom of the flume under pressure, and reappear downstream as transmitted waves.

$2^{\circ} \mathrm{S}$ is completely submerged. The incident waves pass over the sill formed by $\mathrm{s}$ and set up a periodic disturbance, which may be very complex, over and dounstream of $\mathrm{S}$.

It is thus seen that the mork had to be done in two phases and the anthor has presented these parts separately for the reader's convenience.
\end{abstract}

\section{CHAPITRE I. - NATURE DU PROBLÈME}

\section{I. - DEFINITIONS ET HYPOTHËSES}

Considérons un canal indéfini ouvert, à fond lisse et horizontal, à section droite rectangulaire. Une couche de liquide parfait, pesant, de hauteur $h$, au repos, emplit ce canal. Imaginons maintenant qu'on dispose dans le liquide un obstacle $S$ en forme de parallélépipède rectangle, de longueur $l$, de largeur égale à celle du canal et dont la base, supposée horizontale, soit placée à la cote $h_{0}$ par rapport au fond. Le schéma de l'installation ainsi que les axes de coordonnées, qui permettent de repérer les ćléments que l'on vient de définir, sont représentés par la figure 1, qui donne la coupe de l'ensemble par le plan de symétrie vertical.

Ceci étant, supposons qu'une houle irrotationnelle, venant de l'infini négatif, agite le liquide. Nous nous proposons d'étudier l'ensemble du mouvement dans le canal, de préciser, notam- 


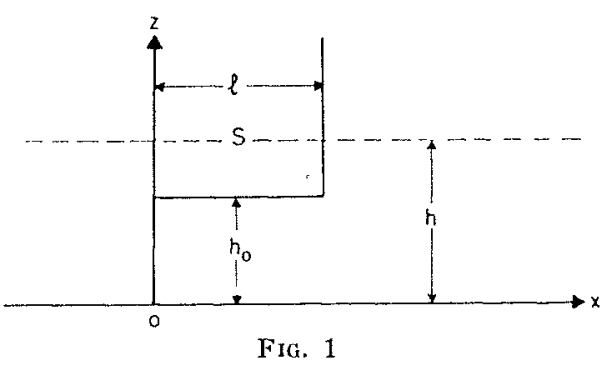

ment, les effets de réflexion de la houle incidente, de déterminer le mouvement du liquide sous l'obstacle et à l'aval de celui-ci. C'est le problème étudié par E.O. Macagno dans [1].

Les mouvements du fluide étant supposés de faible amplitude, les équations de l'hydrodynamique sont linéarisées. Cette approximation ne semble pas trop restrictive. On admet généralement, en effet, que l'influence des termes d'ordre supérieur au premier (par rapport à l'ampli(ude a), est assez faible tant que la cambrure de l'onde ne dépasse pas 0,05 et tant que la profondeur relative $h / L$ est assez grande - supérieure à 0,1 . Toutes les expériences de Macagno ne remplissent pas ces conditions de validité. Il est dès lors très reniarquable que la théorie linéarisée suffise encore à donner une interprétation correcte des expériences. Nous reviendrons sur ce point.
Toutefois, le phénomène se complique, parfois, du fait de la naissance des cavitations dans le voisinage des arêtes immergées de S. Les conditions de naissance de ces décollements sont encore mal élucidées, à notre connaissance, du moins. Elles semblent lices à l'existence d'une amplitude critique, au-delà de laquelle la théorie cadre moins bien avec l'expérience.

En tout état de cause, ces limites de la validité du calcul ne sont mentionnées qu'à titre hypothétique, faute d'avoir une théoric plus satisfaisante du phénomène.

Notre seconde hypothèse fondamentale revient à postuler l'irrotationnalité du mouvement. D'après cela, tout revient à expliciter le potentiel $\Phi^{\prime}$ des vitesses dans le domaine $D$ occupé par le liquide à l'état de repos, e'est-à-dire dans un domaine connu a priori. Enfin, nous supposerons que $\Phi^{\prime}$ est de la forme (complexe) :

$$
\Phi^{\prime}(x, z, t)=\Phi(x, z) e^{(2 \pi i / T) t}
$$

T étant la période unique du phénomène. Autrement dit, nous nous limitons à l'examen du phénomène fondamental. Au reste, le problème étant, comme nous le verrons, non seulement linéaire, mais aussi homogène, la prise en compte des harmoniques serait aisée.

\section{II. - MÉTHODES EMPLOYEES}

Nous avons, en définitive, à déterminer dans le domaine $D$ une fonction harmonique $\Phi(x, z)$, dont il y aura lieu de préciser l'allure à l'infini, satisfaisant aux conditions aux limites suivantes, $\vec{n}$ étant le vecteur unitaire porté par la normale extérieure à $\mathrm{D}$ :

$$
\frac{d w}{d n}=0 \quad \text { pour } \begin{cases}-\infty \leqq x \leqq \infty, z=0 \\ x=0, & h_{0} \leqq z \leqq h \\ x=l, & h_{0} \leqq z \leqq h \\ 0 \leqq x \leqq l, & z=h_{0}\end{cases}
$$

el :

$g \frac{d \Phi}{d n}=\frac{4 \pi^{2}}{\mathrm{~T}^{2}} \Phi \quad$ pour $z=h, \begin{array}{r}-\infty \leqq x \leqq 0 \\ l \leqq x \leqq \infty\end{array}$

On a donc bien affaire à un problème harmonique linéaire et homogène du type mixte. Plusieurs méthodes résolutives sont théoriquement possibles. Yoici, à titre indicatif, le principe de l'une d'entre elles, qui nous a été indiqué par' M. Kravtchenko.

Représentons conformément D sur le cercle unité $|\zeta| \leqq 1$ du plan de la variable complexe; $\zeta=\xi+i \eta=o e^{i n}$; on peut choisir la correspondance $\zeta=\zeta(Z), Z=x+i z$, de manière que les points ci-après soient homologues :

$$
\begin{array}{ll}
\zeta=-1 & Z=-\infty+i z \\
\zeta=1 & Z=+\infty+i z \\
\zeta=-i & Z=(l / 2) \\
\zeta=i & Z=(l / 2)+i h_{0}
\end{array}
$$

La fonction $\zeta(Z)$ peut, d'ailleurs, être explicitée au moyen des formules classiques de SchwarzChristoffel. Posons :

$$
\begin{gathered}
\zeta_{1}=e^{i \alpha_{1}}=\zeta(l+i h), \quad \zeta_{2}=e^{i \alpha_{2}}=\zeta\left(l+i h_{0}\right) ; \\
0 \leqq \alpha_{1} \leqq \alpha_{2} \leqq \pi / 2 .
\end{gathered}
$$

En raison de la symétrie de $\mathrm{D}$ par rapport à la droite $x=l / 2$, les arguments des images des points singuliers $Z=i h_{0}$ et $Z=i h$ du contour de $D$ seront respectivement $\pi--\alpha_{2}$ et $\pi-\alpha_{1}$. Mais on notera que le calcul explicite des paramètres $\alpha_{1}$ et $\alpha_{2}$ en fonction des données $h$ et $h_{0}-$ quoique du type classique - est déjà assez lourd. 
Cela posé, la correspondance $\zeta=\zeta(Z)$ ramène la question à la détermination d'une fonction :

$$
\Phi(\xi, \eta) \equiv \Phi[\xi(x, z), \eta(x, z)]=\Phi(x, z)
$$

harmonique dans $|\zeta| \leqq 1$ et assujettie à vérifier les conditions limites suivantes :

$$
\frac{d \bar{\Phi}}{d n}=0 \quad \text { pour } \quad:=1,\left\{\begin{array}{l}
-\pi \leqq \theta \leqq 0 \\
\alpha_{1} \leqq \theta \leqq \pi-\alpha_{1}
\end{array}\right.
$$

et :

$$
\begin{aligned}
\frac{d \bar{\Phi}}{d n}=g & \left|\frac{d Z}{d \zeta}\right| \bar{\Phi} \\
& \text { pour } \quad \circ=1,0 \leqq 0 \leqq \alpha_{1}, \pi-\alpha_{1} \leqq \theta \leqq \pi
\end{aligned}
$$

Cette méthode de mise en équation du problème offre l'avantage de bien situer la nature analytique de la question et d'en dégager la difficulté, Car tout se trouve être ramené à la détermination dans $\bullet \leqq 1$, de la fonction harmonique $\varphi(\xi, \eta)$, assujettie à vérifier la condition aux limites :

$$
\frac{d \varphi}{d n}+f(\theta) \varphi=0, \quad \varphi=1
$$

où $f(\theta)$ est une fonction donnée a priori pour $0 \leqq 0 \leqq 2 \pi$, nulle, d'ailleurs, sur une portion de $p=1$. Un tel problème aux limites est connu dans la littérature sous le nom de problème de Poincaré; la solution en est bien étudiée dans le cas du cercle, lorsque $f(\theta)$ est une fonction assez régulière de $\theta$ pour $0 \leqq \theta \leqq 2 \pi$, par exemple, suffisamment continue et périodique de période $2 \pi$. Or, dans notre cas, $f(\theta)$ présente, entre autres, des singularités pour $\theta= \pm \pi$. A notre connaissance, une théorie rigoureuse du problème de Poincaré reste encore à faire pour les fonctions $f(\theta)$ du type qui correspond à nos données. Cependant, il y a là le principe d'une méthode de calcul de la solution au moyen des développements en série, valable après avoir opéré la défalcation des singularités; j'espère y revenir avec M. Kravtchenko ultérieurement. La question est d'autant plus intéressante que, comme me l'a fait remarquer M. Kravtchenko, les problèmes des plages inclinées étudiés par MM. Roseau et Brillouet (cf. [10] et [11]) relèvent de la méthode que l'on vient de décrire. Plus généralement, l'étude des petiles oscillations planes du liquide pesant, limité par un fond de forme quelconque, et la surface libre, se ramène à un problème de Poincaré dans le cercle, chaque fois que l'on sait représenter sur ce domaine canonique le domaine occupé par le liquide au repos.

Toutefois, il est douteux que le procédé précédent puisse être perfectionné au point de pouvoir être utilisé en vue du calcul simple et effectif d'une solution. Or, notre objectif est moins l'étude théorique de notre problème que la recherche d'une solution approchée, numériquement calculable. C'est pourquoi nous allons employer la méthode suivante qui conduil assez facilement au but poursuivi, quoiqu'au prix de simplifications non encore justifiées.

Nous décomposons le domaine $\mathrm{D}$ en trois domaines : $D_{1,2}, \quad D_{0}, \quad D_{3}$ définis respectivement comme suit :

$$
\begin{array}{lll}
\mathrm{D}_{1,2} & -\infty \leqq x \leqq 0 & 0 \leqq z \leqq h \\
\mathrm{D}_{0} & 0 \leqq x \leqq l & 0 \leqq z \leqq h_{0} \\
\mathrm{D}_{3} & l \leqq x \leqq \infty & 0 \leqq z \leqq h
\end{array}
$$

En nous inspirant d'une méthode, introduite par A. Apté [2], à la suite d'une suggestion de J. Kravtchenko, nous prenons :

$$
\begin{array}{ll}
\Phi=\Phi_{1}+\Phi_{2} & \text { dans } D_{1.2} \\
\Phi=\Phi_{0} & \text { dans } D_{0} \\
\Phi=\Phi_{3} & \text { dans } D_{3},
\end{array}
$$

où $\Phi_{1}$ est le potentiel créé par la houle incidente; sous forme complexe, on peut donc poser:

$$
\Phi_{1}=\mathbf{A}_{1} \cosh m z e^{-m i x}
$$

où $m=2 \pi / \mathrm{L}$, L étant la longueur d'onde lićc à la période $\mathbf{T}$ de la houle, à l'accélération $g$ de la pesanteur el à $h$ par la relation d'Airy :

$$
k^{2}=(2 \pi / \mathrm{T})^{2}=g m \tanh m h
$$

L'onde en cause étant supposée connue, $A_{1}, m$, et $k$ sont des données.

Le problème consiste maintenant à déterminer les trois fonctions $\Phi_{0}, \Phi_{2}, \Phi_{3}$ harmoniques dans leurs domaines de définition respectifs, astreintes à satisfaire les conditions à la surface libre et de paroi, de manière que $\Phi_{0}$ soit le prolongement analytique de $\Phi_{1}+\Phi_{2}$ (ou de $\Phi_{3}$ ) à travers le segment de droite commun à leurs domaines de définition : $x=0$ (ou $x=-=l$ ), $h_{0} \leqq z \leqq h$.

D'après la remarque d'Apté, il suffit d'exprimer à cet eflet que les expressions considérées de $\Phi$, ainsi que leurs dérivées partielles en $x$, sont identiques le long des segments en cause.

Nous verrons que la question se ramène, en dernière analyse, à la résolution d'un système linéaire à une infinité d'inconnues, que nous nous proposons de discuter ailleurs en toute rigueur. Mais, nous avons formé une solution approchée, en bon accord avec les résultats expérimentaux; et ce sont ces résultats de notre calcul que nous exposons ci-après.

Remarque : Ce mémoire était déjá rédigé lorsque nous avons pu prendre connaissance -grâce à une aimable communication de M. Lattès - du manuscrit d'un travail de MM. Lattès et 
Lions, consacré à des problèmes analogues au nôtre. On trouve dans cette étude une démonstration de la convergence du procédé d'approximations successives d'Apté, problème que nous n'avons pu aborder nous-mêmes ici. Nous espérons revenir sur les conclusions des auteurs précités que nous n'avons pu, faute de lemps, exploiter davantage.

\section{III. - APERÇU DES RESULTATS OBTENUS}

On constatera que, sauf dans une zone transitoire, de faible longueur horizontale, voisine de la face amont (ou aval) de $S$, le potentiel $\Phi_{2}$ (ou $\Phi_{3}$ ) se réduit à celui d'une houle réfléchie (ou transmise) par l'obstacle. Ainsi donc, se trouve confirmés qualitativement et quantitativement les résultats de $[1]$ obtenus au moyen des hypothèses a priori et des raisonnements globaux. Rappelons pour plus de précision les hypothèses de départ de Macagno.

En premier lieu, cet auteur admet que le mouvement dans $\mathrm{D}_{0}$ se réduit à une oscillation sinusoïdale en masse; il postule ensuite que les zones transitoires ont une très faible épaisseur, que l'on peut négliger lors de la mise en équation du problème. Macagno suppose enfin que $\Phi_{2}$ et $\Phi_{3}$ se réduisent aux potentiels des houles linéaires simples. Ceci posé, l'auteur précité écrit l'équation de continuilé pour le débit global à travers les bords verticaux de $D_{0}$ et l'équation des quantités de mouvement pour la masse liquide enfermée dans ce domaine. Il trouve ainsi les expressions, en fonction de $\phi_{1}$, des amplitudes des houles refléchies et transmises ainsi que l'amplitude du mouvement dans $D_{0}$.

Notre analyse est plus précise; elle repose uniquement sur l'hypothese d'existence et d'unicite de la solution en $\Phi$, astreinte à vérifier quelques conditions a l'infini. Alors les hypotheses a priori de Macasno, relatives à l'allure générale de la solution, apparaissent comme conséquences de nos formules résolutives - qui permetlent, par surcroît, d'analyser le mouvement dans les zones transitoires, dont l'étude a été négligée par l'auteur précité.

Il est très remarquable que le calcul de Macagno, si simple et si schématique, conduise à des résultats en bon accord avec l'expérience. Notre théric, tout en ćtant plus satisfaisante au point de vue de la rigueur, améliore, de plus, sur bien des points, cette concordance. Toutefois, ce résultat n'a pu être atteint qu'au prix d'une grande complication des formules résolutives. L'ingénieur continuera done à se servir de la méthode de Macagno. Mais, il nous a paru instructif de justifier, par une analyse un peu plus rigoureuse, les vues rapides de notre prédécesseur.

Insistons encore une fois sur les limites de la validité de la théorie linéaire. Le postulat essentiel en est le suivant : la solution exacte est développable en séries entières, suivant les puissances de la cambrure $A_{1} / L$ de la houle incidente; les séries étant convergentes, pour des valeurs suffisamment petites de ce paramètre. Il s'ensuit que pour $A_{1} / L$ assez petit, la théorie linéaire est valable. A notre connaissance, le postulat n'a été établi jusqu'ici en toute rigueur que dans le cas des ondes progressives, permanentes et périodiques, se progageant en profondeur uniforme $h$; citons les travaux classiques de Levi-Civita, Struick, Mme Dubreil et ceux, plus récents, de R. Gouyon [14]. Ce dernier auleur a montré, de plus, que le rayon de convergence des séries-solutions tend vers zéro avec la profondeur relative, égale au quotient $h / 1$.

Au surplus, sur cet exemple simple, on voit explicitement que les termes d'ordres supérieurs au premier, - ce dernier étant le seul pris en compte par les théories linéaires - tendent vers l'infini pour une cambrure donnée, lorsque $h / \mathrm{L} \rightarrow 0$. On conclut de là que la théorie des ondes progressives ne peut être en bon accord avec l'expérience que si ce dernier paramètre est assez grand. Les mesures faites en laboratoire confirment ces prévisions.

Ainsi donc, l'approximation linéaire de la houle n'est valable, pour les cambrures courantes, que si la longueur d'onde est assez petite vis-à-vis de la profondeur. Il est très probable que cette conclusion vaut encore pour notre phénomène, où la profondeur n'est plus uniforme. Dans l'état actuel de nos connaissances, on ne peut préciser davantage et indiquer les limites numériques de validité à la théorie linéaire. Mais, dans les applications, ce point ne doit jamais être perdu de vue.

Cette remarque est surtout importante dans le cas du schéma étudié dans la deuxième partie de ce travail. 


\section{IV. - INDICATIONS HISTORIQUES}

Le problème abordé ici nous a été posé par M. Kravtchenko et l'étude ci-après a été faite sous sa direction.

Signalons qu'en profondeur infinie, les problèmes de ce genre ont été abordés par W. R. Dean [3] et F. Ursell [4] par d'autres méthodes et sous réserve que l'épaisseur $l$ de $S$ est nulle et la profondeur infinie. Plus récemment, Dmitriev [15] a utilisé, en profondeur finie, une méthode analogue à la nôtre, mais toujours dans le cas particulier où $l=0$.

Le mémoire de Macagno est à l'origine de ce travail. Nous nous en sommes constamment servi tant pour imaginer le schéma de la solution analytique que pour le contrôle expérimental de nos calculs.

Enfin, rappelons une fois encore que la thèse d'Apté nous a été plus d'une fois utile. Nous nous sommes inspirés des procédés de cet auteur pour la mise en équation du problème et pour l'étude des singularités. Des essais de contrôle de la théorie ont été entrepris aux Laboratoires de Mécanique des Fluides de Grenoble par P. Jolas. Signalons enfin que la matière du présent mémoire a été résumée dans deux notes aux C.R.A.S. [5] et [6].

\section{CHAPITRE II. - MISE EN EQUATION DU PROBLÈME}

\section{I. - DIVERSES FORMES DE $\Phi$}

Les hypothèses faites entraînent $\Delta \Phi=0$ dans D. Nous postulerons essentiellement que la solution du problème harmonique aux limites, que nous allons énoncer, existe et est unique : répétons que nous reviendrons ultérieurement sur ce point. Dans ces conditions, il nous suffira de trouver une solution $\Phi$ satisfaisant à l'ensemble des conditions frontières pour affirmer avoir trouvé la solution.

Une des difficultés du sujet tient à la cause suivante: les domaines $D_{1,2}$ et $D_{3}$ ne sont pas bornés et il subsiste un certain arbitraire quant au choix a priori de l'allure de $\Phi$ pour $|x|=\infty$.

Nous admettons que :

$\Phi_{2}(x, z)=A_{2} \cosh m z e^{i m x}+0\left(1 / x^{2}\right)$

pour $x=-\infty$

où $\mathrm{A}_{2}$ est une constante proportionnelle à l'amplitude de la houle incidente. Il résulte alors des travaux de Havelock [7], Biesel [8], Kravtchenko [9], que $\Phi_{2}$ admet dans $D_{1,2}$ un développement en série généralisée de Bessel-Fourier; le développernent formel suivant les termes d'une famille complète de fonctions propres est du type :

$$
\Phi_{2}=A_{2} \cosh m z e^{i m, r}+\sum_{1}^{\infty} B_{n} \cos \sigma_{n} z e^{\sigma_{n} \cdot r}
$$

où $\mathrm{A}_{2}$ et les $\mathrm{B}_{n}$ sont des constantes complexes à déterminer, les $\sigma_{n}$ étant les racines réelles et positives de l'équation transcendante:

$$
k^{2}=-g \sigma_{n} \operatorname{tang} \sigma_{n} h
$$

D'après cela, chaque terme du développement formel (3) est une fonction harmonique, qui vérifie les conditions de fond $\partial \Phi_{2} / \partial z=0$ et de surface libre :

$$
\frac{\partial^{2} \Phi_{2}}{\partial t^{2}}=-g \frac{\partial \Phi_{2}}{\partial z}
$$

Nous laissons de côté pour le moment la condition frontière le long de $x=0,0 \leqq z \leqq h$.

Pour la même raison (et moyennant les mêmes hypothèses quant à l'allure du mouvement pour $x=\infty$ ), on définira $\Phi_{3}$ par le développement :

$\Phi_{3}=A_{3} \cosh m z e^{-i m(x-l)}+\sum_{1}^{\infty} G_{\mathrm{n}} \cos \sigma_{n} z e^{-\omega_{n}(x-1)}$ 
où $A_{3}$ et les $G_{n}$ sont des constantes complexes à déterminer. Cette expression de $\Phi_{3}$ satisfait encore formellement à toutes les conditions imposées, sauf le long de $x=l, 0 \leqq z \leqq h$.

Enfin, dans $D_{0}$, on cherchera $\Phi_{0}$ sous la forme :

$\Phi_{0}=\mathrm{U} x+\mathrm{V}+\sum_{1}^{\infty}\left\{\mathrm{C}_{n} e^{--\lambda_{n} x}+\mathrm{D}_{n} e^{\lambda_{n}(x-l)}\right\} \cos \lambda_{n} z$

où on a posé :

$$
\lambda_{n}=n \pi / h_{g}, \quad n=1,2, \ldots \infty
$$

et ou $\mathrm{U}, \mathrm{V}, \mathrm{C}_{n}$ et $\mathrm{D}_{n}$ sont des constantes, a priori inconnues.

Pour justifier ce mode de représentation de $\Phi_{0}$, il suffit de rappeler que d'après un résultat classique (cf. par exemple [12]), toute fonction harmonique dans le rectangle $\mathrm{D}_{0}$, admettant des dérivées normales nulles le long de $z=h_{0}$ et assez régulières le long de $x=0$ et $x=l$, admet un développement en série généralisée de Bessel-Fourier de la forme :

$\Phi_{0}=\mathrm{U} x+\mathrm{V}+\sum_{1}^{\infty}\left(\mathrm{E}_{n} \cosh \lambda_{n} z+\mathrm{F}_{n} \sinh \lambda_{n} z\right) \cos \lambda_{n} z$ où les quantités $\mathrm{E}_{n}, \mathrm{~F}_{n}$ sont encore des constantes. L'identification de la formule précédente avec (5) est immédiate.

La simple inspection des formules (3) et (4) montre déjà que si ces séries convergent pour $x=0$ et $x=l$ respectivenuent, la somme des séries :

$$
\sum_{1}^{\infty} \mathrm{B}_{n} \cos \sigma_{n} z e^{\sigma_{n} a} \quad \text { et } \quad \sum \mathrm{G}_{n} \cos \sigma_{n} z e^{-\sigma_{n}(x-l)}
$$

tendent très rapidement vers zéro lorsque $x \rightarrow-\infty$ et $x-l \rightarrow \infty$ respectivement. Pratiquement, la somme de la première est négligeable pour $x \leqq--2 h$; (3) montre alors que le mouvement à l'amont se réduit à une faible distance de l'obstacle au clapotis linéaire partiel, engendré par la superposition linéaire des houles de potentiels $\mathrm{A}_{1} \cosh m z e^{-m i x}$ et $\mathrm{A}_{2} \cosh m z e^{m i x}$. La même remarque vaut pour l'aval. Enfin, dans le passage en charge et à une faible distance des extrémités, nous aurons seulement (Cf. [5]) une oscillation sinusoïdale et horizontale en masse, définie par le potentiel $U X+V$. Ainsi se trouvent justifiées a posteriori les hypothèses de Macagno.

\section{II. - DIGRESSION ANALYTIQUE (1)}

Ainsi donc, le problème posé sera entièrement résolu sous forme de séries infinies dès que l'on aura explicité les constantes $A_{2}, A_{3}, U, V, B_{n}, G_{n}$, $\mathrm{C}_{n}, \mathrm{D}_{n}, n=1,2 \ldots \infty$.

Une question de principe se pose : est-il légilime de représenter $\Phi_{0}$, $\Phi_{2}$ et $\Phi_{3}$ au moyen des développements du type précédent. Autrement dit, ces fonctions sont-elles suffisamment régulicres à la frontière (elles le sont, par hypothèse, à l'intérieur de I) pour qu'on puisse être sûr, a priori, de la convergence de nos séries à la surface libre et le long des parois solides. La réponse est affirmative; elle résulte de quelques remarques d'Apté (Cf. [2], pp. 75-78), que nous allons rappeler brièvement.

Supposons tout d'abord démontré que les coefficients $\mathrm{B}_{n}, \mathrm{G}_{n}, \mathrm{C}_{n}$ et $\mathrm{D}_{n}$ tendent vers zéro avec $1 / n$. On voit que pour $x<-\varepsilon<0$, $\varepsilon>0$ étant aussi petit qu'on le veul, la série (3) converge absolument pour $0 \leqq z \leqq h$, puisqu'elle est majorée par la série:

$$
\left|\mathrm{B}_{n}\right| e^{--\boldsymbol{\sigma}_{n} \varepsilon}
$$

(1) Un lecteur qui n'aurait en rue que les applications peut passer ce paragraphe sans inconvénient. visiblement convergente, puisque $\sigma_{n} \sim n \pi / h$. Bien plus, on vérifie qu'il en est de même des séries obtenues en dérivant formellement le second membre de (3) en $x$ et $z$ aulant de fois qu'on le veut. Ainsi, nous pourrons affirmer que :

1. Le développement (3) représente une fonction harmonique dans et sur la fronticre du domaine $D_{1,2, \epsilon}$ :

$$
0 \leqq z \leqq h ; \quad-\infty \leqq x \leqq \varepsilon
$$

aussi petit que soil $\varepsilon>0$.

2. Le second membre de (3) vérifie les conditions frontières au fond et à la surface libre.

Il est clair que le second membre de (4) possède les mêmes propriétés dans le domaine $\mathbf{D}_{3, \epsilon}$ défini par :

$$
x \geqq l+\varepsilon, \quad 0 \leqq z \leqq h
$$

et sur la frontière; la même remarque vaut aussi pour le développement (5) dans le domaine $D_{0, \epsilon}$ :

$$
\varepsilon \leqq x \leqq l-\varepsilon, \quad 0 \leqq z \leqq h_{0} .
$$

Il nous reste donc à examiner ce qui se passe lorsque $\varepsilon \rightarrow 0$. La convergence des séries de Bes- 
sel-Fourier (3), (4) et (5) sur les bords verticaux de leurs domaines de définition respectifs, est essentiellement liée à l'ordre des coefficients $B_{n}$, $\mathrm{G}_{n}, \mathrm{C}_{n}, \mathrm{D}_{n}$ en fonction de $n$. Ici deux voies sont possibles. On peut d'abord chercher à étudier les systèmes linćaires infinis que nous allons expliciler ci-après et que vérifient ces constantes. II faudrait alors déterminer, d'après ces équations de définitions, l'ordre de décroissance de ces inconnues avec $1 / n$ et déduire du résultat une démonstration de la convergence de nos séries pour $\varepsilon=0$. C'est nous semble-t-il, la méthode la plus directe comme la plus naturelle de discussion de notre problème. Malheureusement, sa mise en cuvre est difficile. Dans une prochaine publication, M. Kravtchenko et moi-même établirons seulement les points suivants :

1. La solution du système infini d'équations linéaires est unique.

2. Les séries de termes généraux $\mathrm{B}_{n}{ }^{2}, \mathrm{G}_{n}{ }^{2}, \mathrm{C}_{n}{ }^{2}$, $\mathrm{D}_{n}^{2}$ convergent. Diverses conséquences en résultent, concernant l'allure des seconds membres de (3), (4), et (5) pour $x=0$ et $x=l$ respectivement. Mais, nous n'avons pas réussi encore à fixer l'ordre des coefficients en cause en fonction de $n$, par cette méthode tout au moins.

Nous utiliserons done une méthode indirecte. On sait, en effet, que si la solution $\Phi(x, z)$ de notre problème existe, elle possède les propriétés suivantes :

La fonction $\Phi(x, z)$ est prolongeable analytiquement à travers les segments $h_{0}<z \leqslant h$ $x=0, x=1$; elle est donc régulière sur ces segments, sauf pour $z=h_{0}$, où ses dérivées premic̀res sont infinies comme $\left[x^{2}+\left(z-h_{0}\right)^{2}\right]^{-1 / 3}$ et $\left[(x-l)^{2}+\left(z-h_{0}\right)^{2}\right]^{-1 / 3}$ respectivement. Nous nous dispensons de justifier en détail ces résullats, absolument classiques. Il en résulte que $\Phi$ est fini en les points singuliers, el possède dans chacun de leur voisinage une continuité hölderienne (Cf. Apté, loc. rit.). D'une manière plus précise, on a:

$$
\left|\Phi(x, z)-\Phi\left(0, h_{0}\right)\right| \leqq \mathrm{C}^{\mathrm{te}}\left[x^{2}+\left(z-h_{0}\right)^{2}\right\rceil^{2 / 3}
$$

Il suit de là que :

$$
\begin{aligned}
& \left|\Phi_{2}(0, z)-\Phi_{0}\left(0, h_{0}\right)\right| \leqq \mathrm{C}^{\mathrm{ie}}\left|z-h_{0}\right|^{2 / 3} \\
& \left|\Phi_{0}(0, z)-\Phi_{0}\left(0, h_{0}\right)\right| \leqq \mathrm{C}^{\mathrm{te}}\left|z-h_{0}\right|^{2 / 3}
\end{aligned}
$$

les inégalités analogues étant valables pour $\Phi_{0}$ et $\Phi_{3}$ au point $x==l$ et $z=h_{0}$. Plusieurs corollaires, fondamentaux pour notre objet, en résultent :

1) Les séries (3) et (4) sont absolument et uniformément convergentes pour $x=0$ et $x=l$ respectivement, $0 \leqq z \leqq h$; il en est de même de la série (5) pour $x=0$ et $x=l ; 0 \leqq z \leqq h_{0}$.
2) Les dérivées premières formelles des séries (3) et (4), c'est-à-dire des séries obtenues en dérivant les seconds membres de (3) et (4) terme à terme, par rapport à $x$, convergent uniformément pour $x=0$ el $x=l, 0 \leqq z \leqq h_{0}-\varepsilon$, $h_{0}+\varepsilon \leqq z \leqq h$, aussi petit que soit $\varepsilon>0$; ces sćries sont intégrables lerme à terme sur $0 \leqq z \leqq h$; il en est de même des séries obtenues en dérivant en $x$, terme à terme, le second niembre de (5) pour $x=0, x=l$; les séries en cause convergent uniformément pour :

$$
0 \leqq z \leqq h_{0}-\varepsilon \text {, }
$$

quel que soit $\varepsilon>0$, et sont intégrables terme à terme, pour $0 \leqq z \leqq h_{0}$.

3) L'ordre de grandeur des coefficients $\mathrm{B}_{n}, \mathbf{G}_{n}$, $\mathrm{C}_{n}$ et $\mathrm{D}_{n}$ est $n^{-2 / 3}$, au moins pour $n$ grand.

Ce n'est pas ici le lieu de justifier ces propriétés en toute rigueur et en détail. Nous nous bornerons à de brèves indications, qui seront développées ailleurs.

Tout d'abord, on observera que pour $x=0$ et $x=l$ les expressions de $\Phi_{0}(0, z)$ et $\Phi_{0}(l, z)$ se présentent sous forme d'une série de Fourier en cosinus en $z$ (Cf. [5]); or, on a vu ci-dessus que $\Phi_{0}(0, z)$ et $\Phi_{0}(l, z)$ vérifient une condition de Hölder d'exposant $2 / 3>1 / 2$; d'après le théorème classique de $\mathrm{S}$. Bernstein, les séries de Fourier correspondantes convergent absolument, donc uniformément, pour $0 \leqq z \leqq h_{0}$. Nous renverrons à [13] pour la justification du résultat annoncé relatif à l'expression de $\partial \Phi_{0} / \partial n$, déduite de (5) pour $x=0$ et $x=l$, ainsi que pour l'ordre des coefficients; au reste, ces points peuvent être considérés comme classiques.

Il reste à étendre ces conclusions aux séries (3) et (4) qui ne se réduisent pas pour $x=0$ et $x=l$ à des séries ordinaires de Fourier, en cosinus, puisque les $\sigma_{n} \neq n \pi / h$ : mais on a:

$$
\sigma_{n}=(n \pi / h)+0\left(1 / n^{2}\right)
$$

() $\left(1 / n^{2}\right)$ désignant, suivant l'usage, une quantité d'ordre $1 / n^{2}$. Il s'ensuit que les séries considérées ne difrèrent d'une série de Fourier en cosinus que par une série absolument et uniformément convergente, pour $0 \leqq z \leqq h_{0}$, dérivable une fois en $x$ terme d terme. On justifie alors aisément - quoiqu'au prix de raisonnements un peu fastidieux - les résultats annoncés.

Ainsi donc, nous sommes assurés a priori de la légitimité des opérations que nous avons à effectuer ci-après. Mais plusieurs points de rigueur restent encore en suspens. Par exemple, il faudrait pouvoir vérifier a posteriori que la solution que nous allons construire répond à toutes les conditions qui lui sont imposées. Mais, nous ne saurions insister davantage. 


\section{III. - MISE EN EQUATION DU PROBLEME}

Comme nous l'avons indiqué au paragraphe $3 \mathrm{du}$ chapitre I, il suffit maintenant de déterminer les constantes $\mathrm{A}_{2}, \mathrm{~A}_{3}, \mathrm{U}, \mathrm{V}, \mathrm{B}_{n}, \mathrm{G}_{n}, \mathrm{C}_{n}$ et $\mathrm{D}_{n}, n=1 \ldots \infty$. D'après la remarque d'Apté il faut et il suffit de disposer de ces constantes de manière à satisfaire les relations suivantes :

$$
\begin{array}{cc}
\left.\left(\Phi_{1}+\Phi_{2}\right)\right|_{x=0}=\left.\Phi_{0}\right|_{x=0} & \text { pour } 0 \leqq z \leqq h_{0} \\
\left.\frac{\partial\left(\Phi_{1}+\Phi_{2}\right)}{\partial x}\right|_{x=0} \begin{cases}=\left.\frac{\partial \Phi_{0}}{\partial x}\right|_{x=0} & \text { pour } 0 \leqq z \leqq h_{0} \\
=0 & \text { pour } h_{0} \leqq z \leqq h\end{cases}
\end{array}
$$

et :

$$
\begin{array}{ll}
\Phi_{3} !_{x=l}=\left.\Phi_{0}\right|_{x=l} & \text { pour } 0 \leqq z \leqq h_{0} \\
\left.\frac{\partial \Phi_{3}}{\partial x}\right|_{x=l} \begin{cases}=\left.\frac{\partial \Phi_{0}}{\partial x}\right|_{x=l} & \text { pour } 0 \leqq z \leqq h_{0} \\
=0 & \text { pour } h_{0} \leqq z \leqq h\end{cases}
\end{array}
$$

Toujours d'après l'auteur précité, les conditions nécessaires et suffisantes pour satisfaire (6) peuvent s'écrire :

$$
\begin{gathered}
\int_{0}^{h_{0}}\left[\Phi_{1}+\Phi_{2}\right]_{x=0} \cos \lambda_{n} z d z=\int_{0}^{h_{0}}\left[\Phi_{0}\right]_{x=0} \cos \lambda_{n} z d z \quad n=0,1,2, \ldots \\
\int_{0}^{h}\left[\partial / \partial x\left(\Phi_{1}+\Phi_{2}\right]_{x=0} \cosh m z d z=\int_{0}^{h_{0}}\left[\partial \Phi_{0} / \partial x\right]_{x=0} \cosh m z d z\right. \\
\int_{0}^{h}\left[\partial / \partial x\left(\Phi_{1}+\Phi_{2}\right)\right]_{x=0} \cos \sigma_{n} z d z=\int_{0}^{h_{0}}\left[\partial \Phi_{0} / \partial x\right]_{x=0} \cos \sigma_{n} z d z \quad n=1,2, \ldots
\end{gathered}
$$

De même, les conditions (7) seront vérifiées si :

$$
\begin{gathered}
\int_{0}^{h_{0}}\left[\Phi_{3}\right]_{x=l} \cos \lambda_{n} z d z=\int_{0}^{h_{0}}\left[\Phi_{0}\right]_{x=l} \cos \lambda_{n} z d z \quad n=0,1,2 \ldots \\
\int_{0}^{h}\left[\partial \Phi_{3} / \partial x\right]_{x=l} \cosh m z d z=\int_{0}^{h_{0}}\left[\partial \Phi_{0} / \partial x\right]_{x=0} \cosh m z d z \\
\int_{0}^{h}\left[\partial \Phi_{3} / \partial x\right]_{x=l} \cos \sigma_{n} z d z=\int_{0}^{h_{0}}\left[\partial \Phi_{0} / \partial x\right]_{x=l} \cos \sigma_{n} z d z \quad n=1,2, \ldots
\end{gathered}
$$

Pour expliciter (8) et (9) sous une forme plus condensée, nous poserons :

$$
\begin{aligned}
& \mathrm{I}_{0}=\int_{0}^{h_{0}} \cos ^{2} \lambda_{n} z d z=h_{0} / 2 \\
& \mathrm{I}_{1}=\int_{0}^{h} \cosh ^{2} m z d z=1 / 2\left(h+\frac{\sinh 2 m h}{2 m}\right)
\end{aligned}
$$




$$
\begin{aligned}
& \mathrm{I}_{2^{(n)}}=\int_{0}^{h} \cos ^{2} \sigma_{n} z d z=1 / 2\left(h+\frac{\sin 2 \sigma_{n} h}{2 \sigma_{n}}\right) \\
& I_{3}(n)=\int_{0}^{h_{0}} \cosh m z \cos \lambda_{n} z d z=\frac{(-1)^{n}}{m^{2}+\lambda_{n}^{2}} m \sinh m h_{0}, \\
& \mathrm{I}_{4}^{(n, s)}=\int_{0}^{h_{0}} \cos \sigma_{g} z \cos \lambda_{n} z d z=\frac{(-1)^{n+1}}{\lambda_{n}^{2}-\sigma_{s}^{2}} \sigma_{s} \sin \sigma_{s} h_{0}, \\
& \mathrm{I}_{5}^{(n)}=\int_{0}^{h_{0}} \cos \sigma_{\mathrm{n}} z d z=\frac{\sin \sigma_{n} h_{0}}{\sigma_{n}} \\
& \mathrm{I}_{6}=\int_{0}^{h_{0}} \cosh m z d z=\frac{\sinh m h_{0}}{m} \\
& \mathrm{~T}_{0}=m i \mathrm{I}_{1} h_{0} l+2\left(\mathrm{I}_{6}\right)^{2}+2 m i \mathrm{I}_{1} \sum \frac{\left(\mathrm{I}_{5}(n)\right)^{2}}{\sigma_{2} \mathrm{I}_{2}(n)} \\
& \mathrm{T}^{(n)}=h_{0} l \mathrm{I}_{3}{ }^{(n)}+2 \sum_{s} \mathrm{I}_{5}{ }^{(s)}\left[\mathrm{I}_{3}{ }^{(n)} \mathrm{I}_{5}{ }^{(s)}-\mathrm{I}_{6} \mathrm{I}_{4}{ }^{(n, s)}\right]\left[\sigma_{5} \mathrm{I}_{2}^{(s)}\right]^{-1} \\
& \mathrm{I}_{7}{ }^{(n, s)}=\left[\mathrm{I}_{6}\right]^{-1}\left[\mathrm{~T}_{0}\left(\mathrm{I}_{3}{ }^{(n)} \mathrm{I}_{5}{ }^{(s)}-\mathrm{I}_{6} \mathrm{I}_{4}{ }^{(n, s)}\right)-m i \mathrm{I}_{1} \mathrm{~T}^{(n)} \mathrm{I}_{5}{ }^{(s)}\right]
\end{aligned}
$$

Alors, les conditions (8) et (9) seront respectivement:

$$
\begin{aligned}
& \mathrm{V} h_{0}=\left(\mathrm{A}_{1}+\mathrm{A}_{2}\right) \mathrm{I}_{6}+\sum_{n=1}^{\infty} \mathrm{B}_{n} \mathrm{I}_{5}^{(n)} \\
& \left(\mathrm{C}_{n}+\mathrm{D}_{n} e^{\left.-\lambda_{n} l\right)} \mathrm{I}_{0}=\left(\mathrm{A}_{1}+\mathrm{A}_{2}\right) \mathrm{I}_{3}^{(n)}+\sum_{s=1}^{\infty} \mathrm{B}_{s} \mathrm{I}_{4}{ }^{(n, s)} \quad n=1,2, \ldots \ldots \infty\right. \\
& -m i\left(\mathrm{~A}_{1}-\mathrm{A}_{2}\right) \mathrm{I}_{1}=\mathrm{UI}_{6}-\sum_{n=1}^{\infty} \lambda_{n}\left(\mathrm{C}_{n}-\mathrm{D}_{n} e^{-\lambda_{n}{ }^{l}}\right) \mathrm{I}_{3}^{(n)} \\
& \mathrm{B}_{n} \sigma_{n h} \mathrm{I}_{2}^{(n)}=-\sum_{s=1}^{\infty} \lambda_{s}\left(\mathrm{C}_{s}-\mathrm{D}_{s} e^{-\lambda_{s} 7}\right) \mathrm{I}_{4}^{(s, n)}+\mathrm{UI}_{5_{5}^{(n)}} \quad n=1,2 \ldots \ldots \\
& (\mathrm{U} l+\mathrm{V}) h_{0}=\mathrm{A}_{3} \mathrm{I}_{6}+\sum_{n=1}^{\infty} \mathrm{G}_{n} \mathrm{I}_{5}^{(n)} \\
& \left(\mathrm{C}_{n} e^{-\lambda_{n} l}+\mathrm{D}_{n}\right) \mathrm{I}_{0}=\mathrm{A}_{3} \mathrm{I}_{3}{ }^{(n)}+\sum_{s=1}^{\infty} \mathrm{G}_{n} \mathrm{I}_{4}{ }^{(n, s)} \quad n=1,2, \ldots \ldots \infty \\
& -m i \mathrm{~A}_{3} \mathrm{I}_{1}=\mathrm{UI}_{6}-\sum_{n=1}^{\infty} \lambda_{n}\left(\mathrm{C}_{n} e^{-\lambda_{n} l}-\mathrm{D}_{n}\right) \mathrm{I}_{3}(n) \\
& -\mathrm{G}_{n} \sigma_{n} \mathrm{I}_{2}{ }^{(n)}=\mathrm{UI}_{5_{5}}{ }^{(n)}-\sum_{s=1}^{\infty} \lambda_{s}\left(\mathrm{C}_{s} e^{-\lambda_{s} l}-\mathrm{D}_{s}\right) \mathrm{I}_{4}{ }^{(s, n)} \quad n=1,2 \ldots \ldots \infty
\end{aligned}
$$

Cet ensemble de relations s'écrit encore :

$$
m i \mathrm{I}_{0} \mathrm{I}_{1}\left(1+e^{\left.-\lambda_{n} l\right)} \mathrm{X}_{n}+\sum_{s=1}^{\infty} \lambda_{s} \mathrm{X}_{s}\left(1-e^{\left.-\lambda_{s} l\right)}\left[\mathrm{I}_{3}{ }^{(n)} \mathrm{I}_{3}{ }^{(s)}+m i \mathrm{I}_{1} \sum_{j=1}^{\infty} \frac{\mathrm{I}_{4}{ }^{(s, j)} \mathrm{I}_{4}(n, j)}{\sigma_{j} \mathrm{I}_{2}{ }^{(j)}}\right]=2 \mathrm{~A}_{1} m i \mathrm{I}_{1} \mathrm{I}_{3}{ }^{(n)}\right.\right.
$$




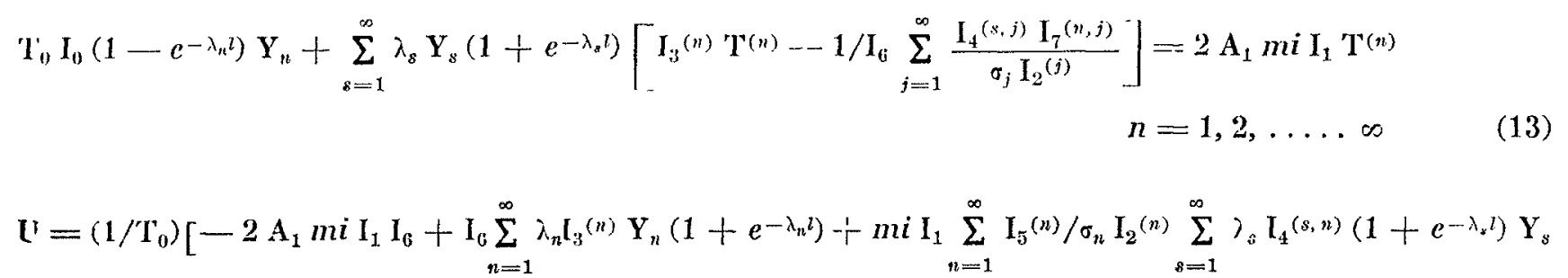

$$
\begin{gathered}
\mathrm{A}_{3}=\left(1 / m i \mathrm{I}_{1}\right)\left[-\mathrm{U} \mathrm{I}_{6}+\sum_{n=1}^{\infty} \lambda_{n}\left(\mathrm{C}_{n} e^{-\lambda_{n} l}-\mathrm{D}_{n}\right) \mathrm{I}_{3}(n)\right. \\
\mathrm{B}_{n}=\left(1 / \sigma_{n} \mathrm{I}_{2}{ }^{(n)}\right)\left[\mathrm{U} \mathrm{I}_{5}^{(n)}-\sum_{s=1}^{\infty} \lambda_{s}\left(\mathrm{C}_{s}-\mathrm{D}_{s} e^{-\lambda_{s} l}\right) \mathrm{I}_{4}^{(s \cdot n)}\right] \\
\mathrm{G}_{n}=\left(1 / \sigma_{n} \mathrm{I}_{2}^{(n)}\right)\left[-\mathrm{U} \mathrm{I}_{5}^{(n)}-\sum_{s=1}^{\infty} \lambda_{s}\left(\mathrm{C}_{s}-e^{-\lambda_{s} l}-\mathrm{D}_{s}\right) \mathrm{I}_{4}^{(s, n)}\right. \\
\mathrm{A}_{2}=\mathrm{A}_{1}+\left(1 / m i \mathrm{I}_{1}\right)\left[\mathrm{U} \mathrm{I}_{6}-\sum_{n=1}^{\infty} \lambda_{n}\left(\mathrm{C}_{n}-\mathrm{D}^{n} \mathrm{e}^{-\lambda_{n} l}\right) \mathrm{I}_{3}{ }^{(n)}\right] \\
\mathrm{V}=\left(1 / h_{0}\right)\left[\left(\mathrm{A}_{1}+\mathrm{A}_{2}\right) \mathrm{I}_{6}+\sum_{n=1}^{\infty} \mathrm{B}_{n} \mathrm{I}_{5}^{(n)}\right],
\end{gathered}
$$

où on a posé :

$$
\begin{aligned}
& \mathrm{X}_{n}=\mathrm{C}_{n}+\mathrm{D}_{n} \\
& \mathrm{Y}_{n}=\mathrm{C}_{n}-\mathrm{D}_{n}
\end{aligned}
$$

Ainsi donc, les équations (11) constituent le systène infini d'équations linéaires relativement aux inconnues $\mathrm{A}_{n}(n>1), \mathrm{B}_{n}, \mathrm{G}_{n}, \mathrm{U}, \mathrm{V}, \mathrm{X}_{n}, \mathrm{Y}_{u}, n=1, \ldots \infty$.

On observera d'ailleur's que tout revient à déterminer en fait les inconnues $\mathrm{X}_{n}$ et $\mathrm{Y}_{n}$ qui figurent seules dans le système (12) et (13). Cette étape franchie, $C_{n}$ et $D_{n}$ sont donnés par (20); les relalions (14) à (19) permettent alors d'exprimer $\mathrm{U}, \mathrm{V}, \mathrm{A}_{2}, \mathrm{~A}_{3}, \mathrm{~B}_{n}, \mathrm{G}_{n}$ au moyen de ces quantités.

Ce n'est pas ici le lieu d'étudier le problème de la résolubilité du système en $\mathrm{X}_{n}$ et $\mathrm{Y}_{n}$. On se bornera à observer qu'il se ramène immédiatement à la forme classique, étudiée par Poincaré, $H$. von Koch, F. Riesz, etc... En sorte que cette question revient à discuter la convergence de certaines séries, formées avec les éléments de la matrice du système linéaire en cause. Au reste, les résultats de ces auteurs ne donnent que des théorèmes d'existence et d'unicité de la solution et permettent de justifier certains processus d'approximations successives de celle-ci. Malheureusement, il n'existe pas - à notre connaissance du moins - de méthode assez bien adaptée à notre cas pour évaluer avec assez de précision l'erreur commise en s'arrêtant à la $n^{\text {ime }}$ approximation. 


\section{IV. - RESOLUTION NUMERIQUE DE SYSTEME EN $\mathbf{X}_{n}$ ET $\mathbf{Y}_{n}$ COMPARAISON AVEC LES EXPERIENCES DE MACAGNO}

D'après ce que nous venons de voir, nous renonçons à utiliser l'appareil théorique de Von Koch pour discuter, au point de vue numérique, le système infini (12) et (13) d'équations en $\mathrm{X}_{n}$ et $\mathrm{Y}_{n}$, auquel nous venons de ramener notre problème. Dans ces conditions, nous opérerons d'une manière très élémentaire, pour ne pas dire brutale : nous remplacerons dans chaque équation en $\mathrm{X}_{n}$ et $\mathrm{Y}_{n}$ les $\underset{1}{\mathrm{\Sigma}}$ par la somme du premier terme et du terme diagonal. O'n verra que dans la gamme des données numériques de Macagno, cette approximation est très suffisante. Mais, on ne saurait trop insister sur le fait que cette manière de faire doit, dans chaque cas particulier, être contrôlée par l'expérience. Il est, en effet, très probable que pour d'autres données numériques, les séries utilisées convergent beaucoup plus lentement et l'approximation qu'on vient d'adopter serait alors médiocre. Au reste, on verra des exemples frappants de ce fait dans la deuxième partie de ce travail.

Rappelons que nous nous bornons dans ce travail uniquement à l'approximation linéaire des équations de l'hydrodynamique; lors même que les termes non linéaires seraient importants, la méthode que nous présentons serait susceptible de donner les lois approchées de passage en charge pour les oscillations fondamentales, de période T. Mais alors, les termes inexplicités ici ne seraient pas négligeables et le phénomène global n'est plus susceptible d'être décrit au moyen de la seule approximation linéaire.

Ceci étant, les équations (12), (13) donnent, si l'on n'y conserve que le premier terme el le terme diagonal :

$$
\begin{aligned}
& \mathrm{X}_{n}=2 \mathrm{~A}_{1} m i \mathrm{I}_{1} \mathrm{I}_{3}^{(n)}\left[m i \mathrm{I}_{0} \mathrm{I}_{1}\left(1+e^{-\lambda_{n} l}\right)+\left.\lambda_{n}\left(1-e^{-\lambda_{n} l}\right)\left\{\left(\mathrm{I}_{3}(n)\right)^{2}+m i \mathrm{I}_{1} \sum_{j=1}^{\infty} \frac{\left.\mathrm{I}_{4}(n, j)\right)^{2}}{\sigma_{j} \mathrm{I}_{2}(j)}\right\}\right|^{-1}\right. \\
& \mathrm{Y}_{n}=2 \mathrm{~A}_{1} \text { mi } \mathrm{I}_{1} \mathrm{~T}^{(n)}\left\lceil\mathrm { I } _ { 0 } \mathrm { T } _ { 0 } \left( 1-e^{\left.-\lambda_{n} l\right)}+\left.\lambda_{n}\left(1+e^{-\lambda_{n} l}\right)\left\{\left(\mathrm{I}_{3}{ }^{(n)}\right) \mathrm{T}^{(n)}--\left(1 / \mathrm{I}_{6}\right) \sum_{j=1}^{\infty} \frac{\mathrm{I}_{4}{ }^{(n, j)} \mathrm{I}_{7}^{(n, j)}}{\sigma_{j} \mathrm{I}_{2}{ }^{(j)}}\right\}\right|^{-1}\right.\right.
\end{aligned}
$$

Ces relations expriment les $\mathrm{X}_{n}$ et $\mathrm{Y}_{n}$ en fonclion des données. Les autres inconnues, déter- minées par (14) à (19), sont alors données par les formules :

$$
\begin{aligned}
& \mathrm{U}=\left(\mathrm{T}_{0}\right)^{-1}\left[-2 \mathrm{~A}_{1} m i \mathrm{I}_{1} \mathrm{I}_{6}+\mathrm{I}_{6} \sum_{n=1}^{\infty} \lambda_{n} \mathrm{I}_{3}{ }^{(n)} \mathrm{Y}_{n}\left(1+e^{\lambda_{n} l}\right)\right] \\
& \mathrm{A}_{2}=\mathrm{A}_{1}+\left(m i \mathrm{I}_{1}\right)^{-1}\left[\mathrm{U} \mathrm{I}_{6}-\sum_{n=1}^{\infty} \lambda_{n}\left(\mathrm{C}_{n}-\mathrm{D}_{n} e^{-\lambda_{n} l}\right) \mathrm{I}_{3}^{(n)}\right] \\
& \mathrm{A}_{3}=\left(m i \mathrm{I}_{1}\right)^{-1}\left[-\mathrm{UI}_{6}+\sum_{n=1}^{\infty} \lambda_{n}\left(\mathrm{C}_{n} e^{-\lambda_{n} l}-\mathrm{D}_{n}\right) \mathrm{I}_{3}^{(n)}\right] \text {, } \\
& \mathrm{G}_{n}=\left[\sigma_{n} \mathrm{I}_{2}^{(n)}\right]^{-1}\left[-\mathrm{U} \mathrm{I}_{5}^{(n)}+\sum_{s=1}^{\infty} \lambda_{s}\left(\mathrm{C}_{s} e^{-\lambda_{s} l}-\mathrm{D}_{s}\right) \mathrm{I}_{4}^{(s, n)}\right] \text {, } \\
& \mathrm{B}_{n}=\left[\sigma_{n} \mathrm{I}_{2}^{(n)}\right]^{-1}\left[\mathrm{U} \mathrm{I}_{5}^{(n)}-\sum_{s=1}^{\infty} \lambda_{s}\left(\mathrm{C}_{s}-\mathrm{D}_{s} e^{-\lambda, l}\right) \mathrm{I}_{4}^{(s, n)}\right] \text {, } \\
& \mathrm{V}=h_{0}^{-1}\left[\left(\mathrm{~A}_{1}+\mathrm{A}_{2}\right) \mathrm{I}_{6}+\sum_{n=1}^{\infty} \mathrm{B}_{n} \mathrm{I}_{5}^{(n)}\right.
\end{aligned}
$$


Dans les applications numériques, nous avons partout remplacé dans les seconds membres des relations précédentes $\sum_{1}^{\infty}$ par $\underset{1}{2}$. Il est bon de dire dès maintenant que cette approximation s'est trouvée être excessive : dans la majorité des cas concrets, la prise en compte du seul premier terme de $\Sigma$ donne, déjà, comme on le verra, une précision très suffisante pour les besoins de la pratique. On trouve dans [1] des données expérimentales relatives aux valeurs des coefficients de transmission $C_{t}$ et de réflexion $C_{r}$, définis par :

$$
\mathrm{C}_{t}=\left|\mathrm{A}_{3} / \mathrm{A}_{1}\right|, \quad \mathrm{C}_{r}=\left|\mathrm{A}_{\mathbf{2}} / \mathrm{A}_{1}\right|,
$$

Ces nombres seuls, au fond, intéressent le technicien. Le lableau ci-après donne les valeurs expérimentales de $\mathrm{C}_{t}, \mathrm{C}_{r}$, empruntées à Macagno et les valeurs théoriques, calculées par le procédé que nous venons de décrire. Nous avons placé entre parenthèses les résultats obtenus en remplaçant les séries des seconds membres de (21) par $\sum_{1}^{2}$ et affecté d'un astérisque les résultats obtenus en prenant en compte $\underset{1}{\stackrel{3}{\Sigma}}$; les nombres qui ne sont affectés d'aucun signe résultent simplement du calcul fait en ne gardant dans (21) que le premier terme des séries qui y figurent.

\begin{tabular}{|c|c|c|c|c|c|c|c|c|c|c|c|}
\hline \multirow{2}{*}{$\begin{array}{c}h \\
(\mathrm{~cm})\end{array}$} & \multirow{2}{*}{$\begin{array}{c}h_{0} \\
(\mathrm{~cm})\end{array}$} & \multirow{2}{*}{$\begin{array}{c}l \\
(\mathrm{~cm})\end{array}$} & \multirow{2}{*}{$\begin{array}{l}\mathrm{T} \\
(\mathrm{s})\end{array}$} & \multicolumn{4}{|c|}{$\mathrm{C}_{t}=\left(\left|\mathrm{A}_{3}\right| \mathrm{A}_{1} \mid\right)$} & \multicolumn{4}{|c|}{$\mathrm{C}_{r}=\left(\left|\mathrm{A}_{2}\right| \mathrm{A}_{1} \mid\right)$} \\
\hline & & & & $\begin{array}{c}\text { Macagno } \\
\text { exp. }\end{array}$ & $\begin{array}{l}\text { Macagno } \\
\text { théorie }\end{array}$ & Takano & Ursell & $\begin{array}{l}\text { Macagno } \\
\text { exp. }\end{array}$ & $\begin{array}{l}\text { Nacagno } \\
\text { théorie }\end{array}$ & Takano & Ursell \\
\hline \multirow[t]{3}{*}{30} & \multirow[t]{3}{*}{15} & \multirow[t]{3}{*}{175} & 1 & $\begin{array}{l}0,067 \\
0,06\end{array}$ & 0,098 & $0,067 *$ & 0,80 & $\begin{array}{l}0,995 \\
0,91\end{array}$ & 0,995 & $1,0^{*}$ & 0,65 \\
\hline & & & 1,4 & $\begin{array}{l}0,134 \\
0,133 \\
0,136\end{array}$ & 0,173 & $\begin{array}{c}0,14 * \\
(0,15)\end{array}$ & 0.98 & $\begin{array}{l}0,98 \\
0,97 \\
0,935\end{array}$ & 0,985 & $\begin{array}{c}0,98 * \\
(0,98)\end{array}$ & 0,15 \\
\hline & & & 1,8 & $\begin{array}{l}0,202 \\
0,178\end{array}$ & 0,239 & 0,20 * & 1,0 & $\begin{array}{l}0,90 \\
0,90\end{array}$ & 0,97 & $0,92 *$ & 0,05 \\
\hline 30 & $\overline{8,5}$ & 175 & 1 & $\begin{array}{l}0,0406 \\
0,036 \\
0,0403\end{array}$ & 0,054 & $\begin{array}{c}(0,043) \\
0,04\end{array}$ & 0,48 & $\begin{array}{l}1,00 \\
0,985 \\
0,935\end{array}$ & 0,998 & $\begin{array}{l}(0,99) \\
1\end{array}$ & 0,86 \\
\hline \multirow[t]{2}{*}{30} & \multirow[t]{2}{*}{4,4} & \multirow[t]{2}{*}{58,3} & 1,3 & 0,476 & 0,635 & $\begin{array}{c}0,59 * \\
(0,59)\end{array}$ & 1 & 0,74 & 0,77 & $\begin{array}{c}0,78^{*} \\
(0,79)\end{array}$ & 0 \\
\hline & & & 1,0 & 0,372 & 0,484 & 0,4 & 0,79 & 0,76 & 0,875 & 0,9 & 0,06 \\
\hline
\end{tabular}

A titre indicalif, nous donnons les valeurs de $\mathrm{C}_{4}$. et $C_{4}$, calculées à partir de la formule de F. Ursel] (Cl. [4] valable, rappelons-le, pour $l=0$. D'un usage lres commode dans la pratique, à cause de sa simplicité, elle donne systématique-

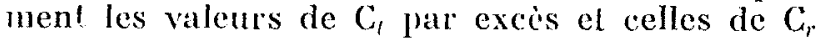
par défaut. Ia théorie variationnelle de la représentation conforme de Lavrentiev permet de prévoir ce fait a prinri.

Voici les remarques que nous a inspirées l'examen du tableau précédent. Dans l'ensemble, nos résultats théoriques cadrent bien avec l'expérience el la concordance du calcul avec les me. sures en canal, est très supérieure à celle que donne la théorie approchée globale de Macagno.

On notera pourtant l'écart sensible qu'on observe entre la théorie et l'expérience pour la période $\mathrm{T}=1,30$. Mais, même dans ce cas, la valeur théorique de $\mathrm{C}_{t}$, évaluée au moyen de notre méthode, est beaucoup plus rapprochée de la valeur expérimentale que ne l'est la valeur de $\mathrm{C}_{t}$ obtenue à partir des formules de Macagno. Par contre, notre valeur du coefficient $C_{\text {r }}$ cadre moins bien avec les mesures que celle de l'auteur précitć.

On notera que cette divergence entre la théorie et l'expérience n'a lieu que pour la plus grande période de la gamme explorée par Macagno. Autrement dit, lors de l'essai correspondant, la hauteur relative du liquide dans le canal est la plus faible. Les remarques antérieures montrent alors que, dans de telles conditions, la convergence de nos séries peut devenir médiocre. 


\section{BIBLIOGRAPHIE}

[1] E. O. Macagno (1954), Houle dans un canal présentant un passage en charge.

La Houille Blanche, $9^{\mathrm{e}}$ année, pp. 10-37.

[2] A.S. APTÉ (1957). Recherches théoriques et expérimentales sur les mouvements de liquides pesants avec surface libre.

Publ. Sci. et Tech. du Ministere de l'Air, $\mathrm{n}^{\circ} 333$.

[3] W. R. Dean (1945). On the reflexion of surface wayes by a sumerged plane barrier. Proc. Camb. Phil. Soc., 41. pp. 231-238.

[4] F. Unsell (1947). The effect of a fixed vertical barrier on surface waves in deep water. Proc. Camb. Soc., 43, pp. 374-382.

[5] K. Takano (1958). Houle dans un canal avec obstacle en charge. C.R.A.S., 246, pp. 3580-3583.

[6] li. Takano (1958). Calcul numérique de la houle dans un canal avec obstacle en charge. C.R.A.S., 247, pp. 673-675.

[7] T.H. Havelock (1929). Forced surface waves on water.

Phil. Mag., 8, pp. 569-576.
[8] F. Biesel (1952). Equations générales au second ordre de la houle irrégulière.

La Houille Blanche, $7^{\mathrm{e}}$ année, pp. 372-376.

[9] J. Kravtchenko (1955). Remarques sur le calcul des amplitudes de la houle linéaire engendrée par un batteur.

Proc. Vth Conf. on Coast. Eng., pp. 50-61.

10] M. Roseat (1952). Contribution à la théorie des ondes liquides de gravité en profondeur variable. Publ. Sci. et Tech. du Ministère de l'Air, $n^{\circ} 275$.

[11] G. Brillouet (1957). Etude de quelques problèmes sur les ondes liquides de gravité. Publ. Sci, et Tech. du Ministère de l'Air, $\mathrm{n}^{\circ} 329$.

[12] L. Kantonovitch et V. KRylov (1952). Méthodes approchées de l'analyse (en russe), Moscou, p. 17.

[13] J. Kravtchenko et A. Apté (1957). Note sur la méthode d'intégration de Fourier des équations de la Physique mathématique.

Annales de l'Institut Fourier, t. IV, pp. 329-358.

[14] R. Gouron (1958). Thèse, Toulouse, sous presse dans les Annales de la Facnlté des Sciences de Toulourse.

[15] A.A. Dmitriev (1959). Passage d'ondes de deux dimensions au-dessus d'un mur noyé. Doklady, pp. 981. 


\title{
DEUXIÈ ME PARTIE
}

\section{EFFET DE PASSAGE DE LA HOULE SUR UN SEUIL}

\author{
C H A P I T R E I \\ POSITION ET MISE EN EQUATION DU PROBLÈME
}

\section{Position du problème.}

Les résultats essentiels de cette partie ont déjà été résumés dans [1].

Les développements de la première partie permettent de simplifier l'exposé de la théorie dont nous allons nous occuper. Les difficultés analytiques sont du même ordre; les énoncés des deux problèmes aux limites sont connus. On verra qu'au point de vue calcul, la différence ne porte que sur la rapidité de convergence des développements en série que nous aurons à utiliser; ce point est d'ailleurs important dans les applications.

Rappelons maintenant le schéma du mouvement. Il s'agit encore de déterminer, dans le cadre de l'approximation linéaire, dans le canal ouvert, comportant un obstacle parallélépipédique S, le mouvement plan irrotationnel et périodique du liquide pesant, se réduisant à l'infini amont à la superposition de deux houles progressives, l'incidente et la réfléchie, et à l'infini aval à la houle trans mise, se propageant dans le même sens que la houle incidente. Mais on suppose maintenant que S, ayant toujours la forme décrite dans la première partie, repose par une de ses faces sur le fond du canal et demeure constamment immergé.

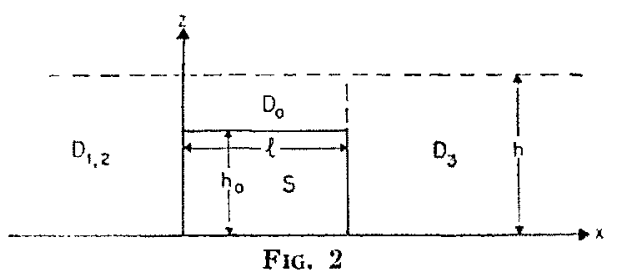

Presque toutes les notations de la première partie demeurent encore valables. Notons, toutefois, que $h_{0}$ désigne ici (Cf. fig. 2) la cote de la face horizontale de $S ; D_{0}$ est maintenant le domaine : $0 \leqq x \leqq l, h_{0} \leqq z \leqq h$, situé au-dessus du seuil S. Pour $l=0$, on obtient la configuration étudiée par Dean [Cf. (7)]. Nous nous proposons encore de définir dans le domaine $\mathrm{D}=\mathrm{D}_{1,2}+\mathrm{D}_{0}+\mathrm{D}_{2,}$, le potentiel des vitesses complexes du type :

$$
\varphi=\Phi(x, z) e^{i(2 \pi / \mathbf{T}) t},
$$

où $\Phi=\Phi_{1}+\Phi_{2}$ dans $D_{1,2}$, à $\Phi_{0}$ dans $D_{0}$, à $\Phi_{3}$ dans $D_{3}$. Les conditions à l'infini et aux limites le long de $z=0$ et $z=h$ comprises à $\varphi$ dans $D_{1,2}$ et $D_{3}$ étant identiques à celles de la première partie, on peut encore écrire :

$$
\begin{aligned}
& \Phi_{1}=A_{1} \cosh m z e^{-m i n} \\
& \Phi_{: z}=A_{2} \cosh m z e^{m i x}+\sum_{1}^{\infty} B_{n} \cos \sigma_{n} z e^{n n} n \\
& \Phi_{3}=A_{3} \cosh m z e^{m i(x-l)}+\sum_{1}^{\infty} \mathbf{G}_{n} \cos \sigma_{n} z e^{-\sigma_{n}(x-l)} \\
& A_{n}=a_{n} e^{i \alpha_{n}}, \ldots
\end{aligned}
$$

en reprenant, pour la commodité du lecteur, les formules (3) et (4). Le potentiel $\Phi_{1}$ sera supposé donné a priori; rappelons que, comme ci-dessus, on a posé :

$$
4 \pi^{2} / \mathrm{T}^{2}=\lambda^{2}=-g \sigma_{n} \text { tang } \sigma_{n} h=-g \lambda^{\prime} \operatorname{tang} \lambda_{n}^{\prime}\left(h-h_{0}\right)
$$


On sait d'autre part (point facile à vérifier directement au moyen de la méthode classique de séparation des variables de Fourier) que toute fonction harmonique assez régulière dans $D_{0}$, vérifiant les conditions de surface libre pour $Z=h$ el de fond pour $Z=h_{0}$, admet le développement de la forme :

$\boldsymbol{\Psi}_{0}=\mathbf{A}_{4} \cosh \mu\left(z-h_{0}\right) e^{i \mu x}+\mathrm{A}_{5} \cosh \mu_{.}\left(z-h_{0}\right) e^{-i \mu x}+\sum_{1}^{\infty}\left\{\mathrm{C}_{n} e^{-\lambda_{n^{\prime}}}+\mathrm{D}_{n} e^{\lambda^{\prime}{ }_{n}(x-l)}\right\} \cos \lambda_{n}^{\prime}\left(z-h_{0}\right)$

où $2 \pi /$ s. désigne la longueur d'onde de la houle linéaire, de la période $T$, se propageant en profondeur ưniforme, égale à $h-h_{0}$. Le problème consiste maintenant à exprimer, par la méthode d'Apté, que ces différentes formes de $\diamond$ sont des prolongements analytiques l'une de l'autre à travers les segments $x=0, x=l, h_{0} \leqq z \leqq h$. On aura ainsi les conditions propres à déterminer les coefficients des développements de $\Phi_{2}, \Phi_{3}, \Phi_{0}$ en fonction de $\Phi_{1}$. On voit encore a priori que les coefficients inconnus $\mathrm{A}_{n}(n \geqq 2), \mathrm{B}_{n}, \mathrm{C}_{n}, \mathrm{D}_{n}(n=1, \ldots \infty)$ seront encore assujettis à vérifier des systèmes d'équations linéaires et homogènes.

Avant de passer aux calculs, notons la parenté de notre régime avec celui de Macagno : les expressions (1) et (2) de $\varphi$ montrent qu'à une faible distance des bords verticaux de $\mathrm{S}$ le mouvement dans $D_{1.2}$ se réduit à un clapotis partiel, résultant de la superposition de la houle incidente et réfléchie et dans $D_{3}$ à la houle transmise.

Enfin, signalons que les singularités du polentiel $\varphi$ le long des arètes de $\mathrm{S}$ de cote $z=h_{0}$ sont identiques à celles étudiées à propos du schéma de Macagno. Le présence de ces points singuliers n'empêche donc pas notre calcul d'être valable. Enfin, les points $x=0, z=0$ et $x=l, z=0$, en lesquels le fond présente des points anguleux, n'introduisent aucune difficulté, le potentiel y étant holomorphe.

\section{Mise en équation du problème.}

Ecrivons maintenant les conditions de raccordement des différentes expressions du potentiel le long des portions communes aux frontières de $D_{1,2}, D_{0}$ et $D_{3}, D_{0}$.

$$
\begin{aligned}
& \Phi_{1}+\Phi_{2}=\Phi_{0} \quad x=0, \quad h_{0} \leqq z \leqq h \\
& \frac{\partial}{\partial x}\left(\Phi_{1}+\Phi_{2}\right)\left\{\begin{array}{lll}
=0 & x=0, & 0 \leqq z \leqq h_{0} \\
=\frac{\partial \Phi_{0}}{\partial x} & x=0, & h_{0} \leqq z \leqq h
\end{array}\right. \\
& \omega_{0}=\Phi_{3} \quad x=l, \quad h_{0} \leqq z \leqq h
\end{aligned}
$$

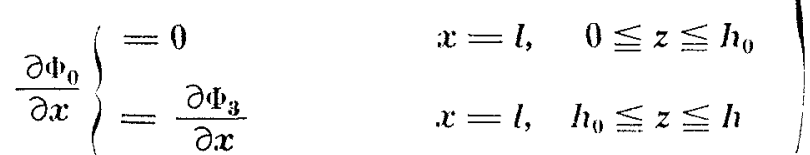

De la même manière que dans la première parlie, les relations (1) à (3) donnent:

$$
\begin{aligned}
& \left(A_{4}+A_{5}\right) I_{7}=\left(A_{1}+A_{2}\right) I_{6}+\sum_{1}^{\infty} B_{n} I_{5}^{(n)}, \\
& \left(\mathrm{C}_{n}+\mathrm{D}_{n} e^{\left.-\lambda_{n}^{\prime} l\right)} \mathrm{I}_{0}^{(n)}=\left(\mathrm{A}_{1}+\mathrm{A}_{2}\right) \mathrm{I}_{3}^{(n)}+\sum_{s=1}^{\infty} \mathrm{B}_{s} \mathrm{I}_{4}^{(n, s)}, \quad(n=1, \ldots \infty)\right. \\
& -m i\left(\mathrm{~A}_{1}-\mathrm{A}_{2}\right) \mathrm{I}_{1}=\mu i\left(\mathrm{~A}_{4}-\mathrm{A}_{5}\right) \mathrm{I}_{6}-\sum_{1}^{\infty} \lambda_{n}^{\prime}\left(\mathrm{C}_{n}-\mathrm{D}_{n} e^{\left.-\lambda^{\prime}{ }_{n} l\right)} \mathrm{I}_{3}(n)\right. \\
& \mathrm{B}_{3 i} \sigma_{n} \mathrm{I}_{2}(n)=\mu i\left(\mathrm{~A}_{4}-\mathrm{A}_{5}\right) \mathrm{I}_{5}^{(n)}--\sum_{s=1}^{\infty} \lambda_{s}^{\prime}\left(\mathrm{C}_{s}-\mathrm{D}_{s} c^{\left.-\lambda^{\prime}, l\right)} \mathrm{I}_{4}^{(s, n)}\right. \\
& (n=1, \ldots \ldots \infty) \\
& \left(\mathrm{A}_{4} e^{\mu i l}+\mathrm{A}_{5} e^{-\mu i l)} \mathrm{I}_{7}=\mathrm{A}_{3} \mathrm{I}_{6}+\sum_{1}^{\infty} \mathrm{G}_{3 i} \mathrm{I}_{5}(n)\right. \\
& \left(\mathrm{C}_{n} e^{-\lambda_{n}^{\prime} l}+\mathrm{D}_{n}\right) \mathrm{I}_{0}^{(n)}=\mathrm{A}_{3} \mathrm{I}_{3}^{(n)}+\sum_{s=1}^{\infty} \mathrm{G}_{s} \mathrm{I}_{4}^{\left(n_{s} s\right)} \\
& (n=1, \ldots \ldots, \infty) \\
& -m i A_{3} I_{1}=\mu i\left(A_{4} e^{\mu i l}-A_{5} e^{-\mu i l}\right) I_{6}-\sum_{1}^{\infty} \lambda_{n}^{\prime}\left(\mathrm{C}_{n} e^{-\lambda_{n}^{\prime} l}-\mathrm{D}_{n}\right) \mathrm{I}_{3}(n) \text {, } \\
& -\mathrm{G}_{n} \sigma_{n} \mathrm{I}_{2}^{(n)}=\mu i\left(\mathrm{~A}_{4} e^{\mu i l}-\mathrm{A}_{5} e^{-\mu i l)} \mathrm{I}_{5}^{(n)}-\sum_{s=1}^{\infty} \lambda_{3}\left(\mathrm{C}_{8} e^{-\lambda^{2}{ }^{2} l}-\mathrm{D}_{8}\right) \mathrm{I}_{4}{ }^{(s, n)}, \quad(n=1, \ldots, \infty)\right.
\end{aligned}
$$


ou :

$$
\begin{aligned}
& \mathrm{I}_{0}{ }^{(n)}=\int_{h_{0}}^{n}\left\{\cos \lambda_{n}^{\prime}\left(z-h_{0}\right) \xi^{2} d z=\frac{1}{2}\left[h-h_{0}+\frac{\sin 2 \ddot{\lambda}_{n}^{\prime}\left(h-h_{0}\right)^{\prime}}{2 \lambda_{n}^{\prime}}\right]\right. \\
& \mathrm{I}_{1}=\int_{0}^{h}(\cosh m z)^{2} d z=\frac{1}{2}\left[h+\frac{\sinh 2 m h^{-}}{2 m}\right] \\
& \mathrm{I}_{2}^{(n)}=\int_{0}^{h}\left(\cos \sigma_{n} z\right)^{2} d z=\frac{1}{2}\left(h+\frac{\sin 2 \sigma_{n} h}{2 \sigma_{n}}\right) \\
& \mathrm{I}_{3}^{(n)}=\int_{h_{0}}^{h} \cos \lambda_{n}^{\prime}\left(z-h_{4}\right) \cosh m z d z \\
& =\frac{\lambda_{n}^{\prime} \sin \lambda_{n}^{\prime}\left(h-h_{0}\right) \cosh m h+m\left\{\cos \lambda_{n}^{\prime}\left(h-h_{0}\right) \sinh m h-\sinh m h_{0}\right\}}{\lambda_{n}^{\prime}+m^{2}} \\
& I_{4}^{(n, s)}=\int_{h_{0}}^{h} \cos \sigma_{g} z \cos \ddot{\lambda}_{n}^{\prime}\left(z-h_{0}\right) d z \\
& =\frac{\sigma_{s}\left\{\cos \lambda_{n}^{\prime}\left(h-h_{0}\right) \sin \sigma_{s} h-\sin \sigma_{s} h_{0}\right\}-\lambda_{n}^{\prime} \sin \lambda_{n}^{\prime}\left(h-h_{0}\right) \cos \sigma_{s} h}{\sigma_{s}^{2}-\lambda_{n}^{\prime}{ }^{2}} \\
& \mathrm{I}_{5}(n)=\int_{h_{0}}^{h} \cos \sigma_{n} z \cosh \mu\left(z-h_{0}\right) d z \\
& =\frac{\sigma_{n}\left\{\sin \sigma_{n} h \cosh \mu\left(h-h_{0}\right)-\sin \sigma_{n} h_{0}\right\}+\mu \cos \sigma_{n} h \sinh \mu\left(h-h_{0}\right)}{\sigma_{n}^{2}+\mu^{2}} \\
& \mathrm{I}_{6}=\int_{h_{0}}^{h} \cosh m z \cosh \mu\left(z-h_{0}\right) d z \\
& =\frac{m\left\{\sinh m h \cosh \mu\left(h-h_{0}\right)-\sinh m h_{0}\right\}-\mu \cosh m h \sinh \mu\left(h-h_{0}\right)}{m^{2}-\mu^{2}} \\
& \mathrm{I}_{7}=\int_{h_{0}}^{h}\left\{\cosh \mu\left(z-h_{0}\right)\right\}^{2} d z=\frac{1}{2}\left[h-h_{0}+\frac{\sinh 2 \mu\left(h-h_{0}\right)}{2 \mu}\right]
\end{aligned}
$$

Après avoir éliminé $\mathrm{A}_{n}(n \geqq 2) \mathrm{B}_{n}, \mathrm{G}_{n}(n \geqq 1)$, on obtient :

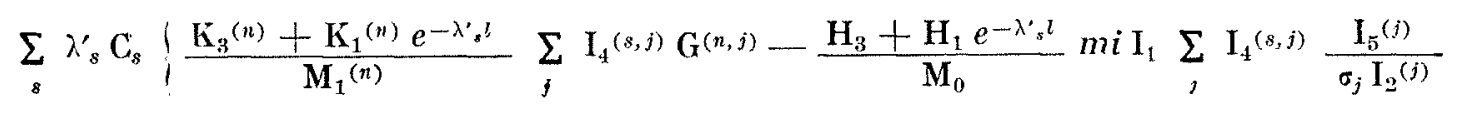

$\left.-\frac{\mathrm{I}_{6} \mathrm{I}_{3}^{(s)}}{\mathbf{M}_{0}}\left(\mathrm{H}_{3}+\mathrm{H}_{1} e^{\left.-\lambda_{s}^{\prime} l\right)}\right\}-\sum_{s} \lambda_{s}^{\prime} \mathrm{D}_{s}\right\} \frac{\mathrm{K}_{3}^{(n)} e^{-\lambda^{\prime} s^{l}}+\mathrm{K}_{1}^{(n)}}{\mathbf{M}_{1}^{(n)}} \sum_{j} \mathbf{I}_{4}^{(s, j)} \mathbf{G}^{(n, j)}-\frac{\mathrm{H}_{3} e^{-\lambda_{s}^{\prime} l}+\mathrm{H}_{1}}{\mathbf{M}_{0}} \boldsymbol{m i} \mathrm{I}_{1}$

$\times \sum_{j} \mathrm{I}_{4}^{(s, j)} \frac{\mathrm{I}_{5}^{(j)}}{\sigma_{j} \mathrm{I}_{2}(j)}-\frac{\mathrm{I}_{6} \mathrm{I}_{3}^{(s)}}{\mathbf{M}_{0}}\left(\mathrm{H}_{3} e^{-\lambda_{s} l}+\mathrm{H}_{1}\right)$

$-\frac{\mathrm{I}_{0}^{(n)} \mathrm{I}_{6}}{\mathrm{M}_{1}{ }^{(n)}}\left\{\mathrm{C}_{n}\left(\mathrm{~K}_{3}{ }^{(n)}-\mathrm{K}_{1}{ }^{(n)} e^{\left.-\lambda_{n}^{\prime}{ }^{l}\right)}-\mathrm{D}_{n}\left(\mathrm{~K}_{1}{ }^{(n)}-\mathrm{K}_{3}{ }^{(n)} e^{\left.-\lambda_{n}^{\prime} l\right)}\right\}=-\frac{2 \mathrm{~A}_{1} m i \mathrm{I}_{1} \mathrm{I}_{6} \mathrm{H}_{3}}{\mathrm{M}_{0}} \quad n=1, \ldots \infty\right.\right.$

$\left.\sum_{\delta} \lambda_{s}^{\prime} \mathrm{C}_{s}\right)\left(\frac{\mathrm{H}_{3}}{\mathrm{M}_{2}{ }^{(n)}}-\frac{\mathrm{K}_{3}^{(n)}+\mathrm{K}_{1}^{(n)} e^{-\lambda_{n^{\prime}} t}}{\mathrm{M}_{1}(n)}\right) \sum_{j} \mathrm{I}_{4}^{(s, j)} \mathrm{G}^{(n, j)}+e^{-\lambda^{\prime} l} \frac{m i \mathrm{I}_{1} \mathrm{~K}_{1}^{(n)}}{\mathrm{M}_{2}{ }^{(n)}} \sum_{j} \mathrm{I}_{4}^{(s, l)} \frac{\mathrm{I}_{5}^{(j)}}{\sigma_{j} \mathrm{I}_{2}^{(j)}}$

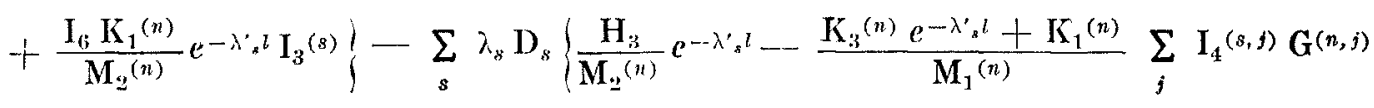

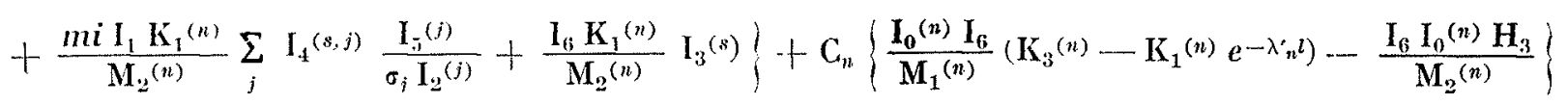

$$
\begin{aligned}
& -\mathrm{D}^{n}\left\{\frac { \mathrm { I } _ { 0 } ^ { ( n ) } \mathrm { I } _ { 6 } } { \mathrm { M } _ { 1 } { } ^ { ( n ) } } \left(\mathrm{~K}_{1}{ }^{(n)}-\mathrm{K}_{3}{ }^{(n)} e^{\left.-\lambda_{n}^{\prime}\right)}+\frac{\mathrm{I}_{6} \mathrm{I}_{0}^{(n)} \mathrm{H}_{3}}{\mathrm{M}_{2}(n)}=0 \quad n=1, \ldots \ldots \infty,\right.\right.
\end{aligned}
$$

$\mathrm{A}_{i}=\frac{2 \mathrm{~A}_{1} m i \mathrm{I}_{1} \mathrm{I}_{6} \mathrm{H}_{3}}{\mathrm{M}_{0}}-\frac{1}{\mathrm{M}_{0}}\left[\sum_{n} \mathrm{H}_{3}\left(\mathrm{C}_{n}-\mathrm{D}_{n} e^{\left.-\lambda_{n l}^{\prime} l\right)}\left\{\mathrm{I}_{6 i} \lambda_{n}^{\prime} \mathrm{I}_{3}{ }^{(n)}+m i \mathrm{I}_{1} \lambda_{n}^{\prime} \sum_{s} \frac{\mathrm{I}_{5}^{(8)}}{\sigma_{s} \mathrm{I}_{2}{ }^{(s)}} \mathrm{I}_{4}{ }^{(n, s)}\right\}\right.\right.$

$$
\left.+\sum_{n} \mathrm{H}_{1}\left(\mathrm{C}_{n} e^{-\lambda_{n}^{\prime} l}-\mathrm{D}_{n}\right)\left\{\mathrm{I}_{6} \lambda_{n}^{\prime} \mathrm{I}_{3}(n)+m i \mathrm{I}_{1} \lambda_{n} \sum_{s} \frac{\mathrm{I}_{5}(s)}{\sigma_{8} \mathrm{I}_{2}{ }^{s}} \mathrm{I}_{4}{ }^{(n, s)}\right\}\right]
$$




$$
\begin{aligned}
& \mathrm{A}_{4}=\left(1 / \mathrm{H}_{1}\right)\left[2 \mathrm{~A}_{1} m i \mathrm{I}_{3} \mathrm{~J}_{6}-m i \mathrm{I}_{1} \sum_{n} \frac{\mathrm{I}_{5}{ }^{(n)}}{\sigma_{n} \mathrm{I}_{2}{ }^{(n)}} \sum_{s} \lambda_{s}^{\prime}\left(\mathrm{C}_{s}-\mathrm{D}_{s} e^{\left.-\lambda_{s}^{\prime}\right)} \mathrm{I}_{4}^{(s, n)}\right.\right. \\
& \left.--\mathrm{I}_{6} \sum_{s} \lambda_{s}^{\prime}\left(\mathrm{C}_{8}-\mathrm{D}_{s} e^{-\lambda_{s}^{\prime} l}\right) \mathrm{I}_{3}^{(s)}-\mathrm{A}_{5} \mathrm{H}_{2}\right] \\
& \mathrm{A}_{3}=-\frac{1}{m i \mathrm{I}_{1}}\left[\mu i\left(\mathrm{~A}_{4} e^{\mu i l}-\mathrm{A}_{5} e^{-\mu i l}\right) \mathrm{I}_{6}-\sum_{n} \lambda_{n}^{\prime}\left(\mathrm{C}_{n} e^{-\lambda_{n}{ }^{l}-}-\mathrm{D}_{n}\right) \mathrm{I}_{3}^{(n)}\right] \\
& \mathrm{A}_{2}=\mathrm{A}_{1}+\frac{1}{m i \mathrm{I}_{1}}\left[\mu,\left(\mathrm{A}_{4}-\mathrm{A}_{5}\right) \mathrm{I}_{6}-\sum_{n} \lambda_{n}\left(\mathrm{C}_{n}-\mathrm{D}_{n} e^{\left.-\lambda_{n}^{\prime}\right) \mathrm{I}_{3}^{(n)}}\right]\right. \\
& \mathrm{B}_{n}=\frac{1}{\sigma_{n} \mathrm{I}_{2}^{(n)}}\left[\mu i\left(\mathrm{~A}_{4}-\mathrm{A}_{5}\right) \mathrm{I}_{5}^{(n)} \cdot-\sum_{s} \lambda_{8}^{\prime}\left(\mathrm{C}_{s}-\mathrm{D}_{s} e^{-\lambda_{s} l}\right) \mathrm{I}_{4}^{(s, n)}\right. \\
& \mathrm{G}_{n}=\frac{1}{\sigma_{u} \mathrm{I}_{2}^{(n)}}\left[-\mu i\left(\mathrm{~A}_{4} e^{\mu i l}-\mathrm{A}_{5} e^{\mu i l}\right) \mathrm{I}_{5}^{(n)}+\sum_{s} \lambda_{8}^{\prime}\left(\mathrm{C}_{8} e^{-\lambda^{\prime} s}-\mathrm{D}_{8}\right) \mathrm{I}_{4}^{\left(s_{1} n\right)}\right]
\end{aligned}
$$

où :

$$
\begin{aligned}
& \mathrm{K}_{1}^{(n)}=\mathrm{I}_{\mathrm{i}} \mathrm{I}_{3}^{(n)}-\mathrm{T}^{(n)}, \\
& \mathrm{K}_{2}{ }^{(n)}=\mathrm{I}_{7} \mathbf{I}_{3}(n)-\frac{1}{1} \mathrm{~T}^{(n)} \\
& \mathrm{K}_{3}{ }^{(n)}=\left(\mathrm{I}_{\boldsymbol{i}} \mathrm{I}_{3}^{(n)}+\mathrm{T}^{(n)}\right) e^{\mu i l} \text {, } \\
& \mathrm{K}_{4}{ }^{(n)}=\left(\mathrm{I}_{7} \mathrm{I}_{3}^{(n)}-\mathrm{T}^{(n)}\right) e^{-\mu i l} \\
& \mathrm{M}_{0}=\mathrm{H}_{2} \mathrm{H}_{3}-\mathrm{H}_{1} \mathrm{H}_{4} \text {, } \\
& \mathrm{M}_{1}{ }^{(n)}=\mathrm{K}_{2}{ }^{(n)} \mathrm{K}_{3}{ }^{(n)}-\mathrm{K}_{1}^{(n)} \mathrm{K}_{4}{ }^{(n)} \text {, } \\
& \mathrm{M}_{2}{ }^{(n)}=\mathrm{H}_{3} \mathrm{~K}_{2}{ }^{(n)}-\mathrm{H}_{4} \mathrm{~K}_{1}{ }^{(n)} \text {, } \\
& \mathrm{T}^{(n)}=\mu . i \sum_{s} \frac{\mathrm{I}_{3}{ }^{(n)} \mathrm{I}_{5}{ }^{(s)}-\mathrm{I}_{6} \mathrm{I}_{4}^{(n, s)}}{\sigma_{s} \mathrm{I}_{2}^{(s)}} \mathrm{I}_{5}^{(s)} \\
& \mathrm{R}=\mu i \sum_{s} \frac{\left(\mathrm{I}_{5}^{(s)}\right)^{2}}{\sigma_{s} \mathrm{I}_{g}(s)} \\
& \mathrm{H}_{1}=m i \mathrm{I}_{1} \mathrm{I}_{\tau}-\mu i\left(\mathrm{I}_{6}\right)^{2}-m i \mathrm{I}_{1} \mathrm{R} \text {, } \\
& \mathrm{H}_{2}=m i \mathrm{I}_{1} \mathrm{I}_{7}+\mu i\left(\mathrm{I}_{6}\right)^{2}+m i \mathrm{I}_{1} \mathrm{R} \text {, } \\
& \mathrm{H}_{3}=\left\{m i \mathrm{I}_{1} \mathrm{I}_{\tau}+\mu i\left(\mathrm{I}_{6}\right)^{2}+m i \mathrm{I}_{1} \mathrm{R}\right\} e^{\mu i l} \text {, } \\
& \mathrm{H}_{4}=\left\{m i \mathrm{I}_{1} \mathrm{I}_{7}-\mu i\left(\mathrm{I}_{6}\right)^{2}-m i \mathrm{I}_{1} \mathrm{R}\right\} e^{-\mu i l} \text {, } \\
& \mathbf{G}^{(n, s)}=\frac{\mathrm{I}_{3}^{(n)} \mathrm{I}_{5}^{(s)}-\mathrm{I}_{6} \mathrm{I}_{4}^{(n, s)}}{\pi_{8} \mathrm{I}_{2}^{(s)}}
\end{aligned}
$$

Le système (4) obtenu ci-dessus définit les constantes inconnues $\mathrm{A}_{n}(n \geqq 2), \mathrm{B}_{n}, \mathrm{C}_{n}, \mathrm{D}_{n}(n=1, \ldots \infty)$ en fonction de $A_{1}$. Une simple inspection des formules montre que les relations obtenues sont beaucoup plus complexes que celles relatives au cas de Macagno. Cela tient essentiellement au fait que le mouvement dans $D_{0}$ ne se réduit plus à une simple oscillation sinusoïdale en masse (excepté le voisinage des segments $x=0$ et $x=I$ ). Il faut donc s'attendre à ce que la convergence du procédé approché que nous allons appliquer pour résoudre le système infini précédent, soit beaucoup plus lente.

Au point de vue pratique, nous observerons que les équations (5) et (6) suffisent à définir les $C_{n}$ et $D_{n}$ en fonction de $A_{1}$; mais cette fois, ces relations sont beaucoup moins symétriques que celles relatives au cas de Macagno et ne permettent plus d'introduire les inconnues auxiliaires $\mathbf{X}_{n}$ et $\mathbf{Y}_{n}$ (Cf. (12) et (13) de la première partie). Les $C_{n}$ et $D_{n}$ étant calculés, les conditions (7) à (12) donnent les autres inconnues dans l'ordre: $A_{5}$ et $A_{4}, A_{2}$ et $A_{3}, B_{n}$ et $G_{n}$. 


\section{H A P I T RE II}

\section{RÉSOLUTIONS NUMERIQUES DU PROBLEME DU SEUIL; CONTROLE EXPERIMENTAL. VALIDITE DE LA METHODE}

\section{Résolution numérique du système linéaire infini. Contrôle expérimental.}

Nous avons encore laissé de côté la discussion de l'existence et d'unicité des solutions du système infini auquel se ramène le problème du seuil. On se bornera donc à l'emploi d'une méthode bruLale; on supprimera dans les $\sum_{1}^{\infty}$ qui figurent aux seconds membres de nos équations les termes supérieurs à un entier donné $\mathrm{N}$, ne dépassant pas 3. Pour tester expérimentalement la rapidité de la convergence, nous avons pris successivement $\mathrm{N}=0,1,2,3$. Les données numériques choisies sont relatives aux expériences de $P$. Jolas [Cf.. (2)], poursuivies aux Laboratoires de Mécanique des Fluides de Grenoble:

$$
h=31,2 \mathrm{~cm} ; \quad h_{0}=20,0 ; \quad l=100,8 \mathrm{~cm}
$$

Les périodes retenues pour le calcul forment la suite :

$$
0,8 \mathrm{~s} ; 0,9 \mathrm{~s} ; 1,0 \mathrm{~s} ; 1,1 \mathrm{~s}
$$

elles diffèrent très peu des périodes utilisées par Jolas :

$$
0,816 \mathrm{~s} ; \quad 0,910 \mathrm{~s} ; 1,005 \mathrm{~s} ; 1,104 \mathrm{~s}
$$

Les valeurs numériques de $T$ prises pour les calculs sont commodes pour l'exécution de ceuxci; et l'écart entre nos résultats théoriques et les mesures expérimentales de Jolas est malheureu-

\begin{tabular}{|c|c|c|c|c|c|c|c|c|c|c|c|c|c|c|c|c|c|}
\hline \multicolumn{3}{|c|}{ EXPÉRIENCE } & & \multicolumn{4}{|c|}{ SOLUTION GLOBALE } & \multicolumn{8}{|c|}{ SOLUTION TIÉORIQUE } & & \\
\hline \multirow{2}{*}{$\mathbf{T}$} & \multirow{2}{*}{$\mathrm{C}_{r}$} & \multirow{2}{*}{$\mathrm{C}_{t}$} & \multirow{2}{*}{$\mathrm{T}$} & \multicolumn{2}{|c|}{$S_{1}$} & \multicolumn{2}{|c|}{$\mathrm{S}_{2}$} & \multicolumn{2}{|c|}{$\mathrm{N}=0$} & \multicolumn{2}{|c|}{$N=1$} & \multicolumn{2}{|c|}{$\mathbf{N}=2$} & \multicolumn{2}{|c|}{$N=3$} & \multicolumn{2}{|c|}{ Dean } \\
\hline & & & & $\mathrm{C}_{r}$ & $\mathrm{C}_{t}$ & $\mathrm{C}_{r}$ & $\mathrm{C}_{\ell}$ & $\mathrm{C}_{r 0}$ & $\mathrm{C}_{t 0}$ & $\mathrm{C}_{r 1}$ & $\mathrm{C}_{i 1}$ & $\mathrm{C}_{r^{2}}$ & $\mathrm{C}_{t 2}$ & $\mathrm{C}_{r 3}$ & $\mathrm{C}_{t 3}$ & $\mathrm{C}_{r}$ & $\mathrm{C}_{t}$ \\
\hline 0,816 & 0,040 & 0,910 & 0,800 & 0,477 & 0,879 & 0,173 & 0,985 & 0,141 & 1,088 & 0,091 & 0,991 & 0,070 & 0,995 & 0,079 & 0,997 & - & - \\
\hline 0,910 & 0,177 & 0,877 & 0,900 & 0,568 & 0,824 & 0,281 & 0,960 & 0,125 & 0,993 & 0,214 & 0,979 & 0,640 & 0,972 & 0,299 & 0,972 & - & 一 \\
\hline 1,005 & 0,222 & 0,842 & 1,000 & 0,177 & 0,984 & 0,091 & 0,996 & 0,028 & 1,012 & 0,283 & 1,007 & 0,778 & 1,127 & 0,780 & 1,122 & 0,30 & 0,97 \\
\hline 1,104 & 0,110 & 0,850 & 1,100 & 0,270 & 0,963 & 0,161 & 0,987 & 0,102 & 0,990 & 0,173 & 0,965 & 0,773 & 0,992 & 0,797 & 1,012 & 0,35 & 0,94 \\
\hline
\end{tabular}
sement trop élevé pour pouvoir être attribué à l'écart des données relatives aux périodes.
Pour $\mathrm{N}=0$ et $\mathrm{N}=1$, nous avons effectué les calculs par les équations (5) à (12) avec une machine digitale; pour $\mathrm{N}=2$ et $\mathrm{N}=3$, nous avons utilisé pour le système (4) la machine du Laboratoire de Calcul de l'Institut Polytechnique de Grenoble.

Comme dans le cas de Macagno, la confrontalion de la théorie et de l'expérience a porté sur les coefficients de transmission $C_{t}$ et de réflexion $\mathrm{C}_{r}$, définis respectivement par :

$$
\mathrm{C}_{t}=a_{2} / a_{1} ; \quad \mathrm{C}_{r}=a_{3} / a_{1}
$$

Nous noterons $C_{t \mathrm{Y}}$ et $\mathrm{C}_{\mathrm{r} \mathrm{x}}$ les valeurs de ces coefficients calculées pour un $\mathrm{N}$ donné; dans le tableau résumant nos résultats, les nombres ainsi obtenus figurent dans la colonne : solution théorique.

Une difficulté pratique est apparue au cours de l'exécution des calculs à la machine. Chacune des constantes inconnues $\mathrm{A}_{n}, \mathrm{~B}_{n}$, etc., étant complexe, équivaut à deux inconnues réelles; le programme de la machine du Laboratoire de Calcul n'étant valable que pour les systèmes réels, on voit que pour $\mathrm{N}=3$, nous avons à résoudre (4) en système de 32 équations linéaires. En tout état de cause, la convergence des approximations successives ne pouvait pas être rapide.

L'examen du tableau des résultats montre que pour $\mathrm{T}=0,8 \mathrm{~s}$, la convergence des $\mathrm{C}_{r \mathrm{~N}}$ et $\mathrm{C}_{t \mathrm{~N}}$ est relativement satisfaisante. Sauf pour $\mathrm{N}=3$, 
$\mathrm{C}_{r \mathrm{~N}}$ tend d'une manière monotone vers la valeur expérimentale; mais l'écart entre la théorie et les mesures reste élevé, de l'ordre de $50 \%$. Les résultats sont bien meilleurs en ce qui concerne $\mathrm{C}_{t ঙ}$. Pour les autres périodes, la concordance entre les calculs et l'expérience devient médiocre; à noter que l'allure des $C_{r X}$ et $C_{t N}$, en tant que fonctions de $\mathrm{N}$, devient de plus en plus oscillante et l'écart entre les valeurs théoriques et expérimentales de ces coefficients augmente avec $T$. Nous tenterons, un peu plus loin, de donner une interprétation théorique de ces conclusions qualitatives. Quoi qu'il en soit, il ne semble pas possible de demander au calcul autre chose que l'ordre de grandeur du phénomène, tout au moins pour la gamme des données numériques cidessus. C'est pourquoi nous avons tenté d'appliquer à l'étude du passage de la houle sur un seuil quelques-unes des formules semi-empiriques, d'un emploi beaucoup plus commode, ohtenues à partir des raisonnements globaux, analogues à ceux de Macagno. Signalons, dans cet ordre d'idées, les travaux de H. Jeffreys [Cf. (3) ], Le Méhauté [Cf. (4)], Valembois [Cf. (5)]. Nous avons nous-même obtenu quelques résultats analogues, en suivant la voie inaugurée par Lamb [Cf. (6)]. A cet effet, nous adoptons l'hypothèse hydraulique des tranches (ce qui revient à postuler que pour une abscisse $x$ donnée, la réparlition des vitesses est horizontale et uniforme) et nous exprimons ensuite la continuité des débits et des pressions le long des frontières communes aux domaines $D_{1.2}$ et $D_{0}, D_{0}$ et $D_{3}$. Nous avons tenté ensuite d'améliorer ces hypothèses, un peu grossières, en admettant a priori que pour lout $x$, la répartition des vitesses en $z$ étail donnée par une fonction hyperbolique. Nous nous bornons ici à donner le résultat de notre calcul approché :

$$
\begin{aligned}
& A_{4}=\frac{2(1-\delta) A_{1}}{(1-\delta)^{2}-(1+\delta)^{2} e^{2 \mu / i}} \\
& A_{5}=-\frac{1+\delta}{1-\delta} e^{2 \mu h} A_{4}, \\
& A_{3}=-\frac{2 \delta}{1-\delta} e^{\mu l} A_{4} \\
& A_{3}=A_{5}+A_{4}-A_{1},
\end{aligned}
$$

où :

$$
\delta=(m / \mu)
$$

Les applications numériques figurent dans le tableau sous la rubrique : Théorie globale; les formules de Jeffreys. Valembois, etc., donnent des résultats voisins qui ne sont pas, dès lors, mentionnés explicitement. On voit que la concordance avec les résultats numériques est médiocre; elle est, d'ailleurs, moins bonne que celle donnée par les approximations des divers ordres de notre thcorie exacte pour les petites valeurs de $T$; les résultats sont en gros, inverses pour $T$ grand. Nous reviendrons ci-dessus sur ces points. Enfin, nous avons ajouté à notre tableau les valeurs des coefficients calculés à partir de la solution de Dean [Cf. (7)], exacte pour $l=0$ et $h=\infty$; on peut apprécier ainsi l'influence du paramètre $l$.

\section{Validité du calcul approché.}

La théorie du calcul approché des effets de passage d'une houle sur le seuil n'est pas assez avancée à l'heure actuelle et les données expérimentales disponibles trop rares pour qu'on puisse tenter une interprétation sûre des écarts constatés entre nos prévisions théoriques, d'une part, et les résultats des mesures, d'autre part. Les réflexions qui suivent ne sont donc présentées que sous toutes réserves, avec le seul espoir de guider utilement les futures recherches sur ce sujet difficile.

Plaçons-nous d'abord au point de vue purement analytique. Supposons que les équations linéarisées représentent convenablement le phénomène, sans nous préoccuper de la limite de la validité physique de cette hypothèse. Dans cette éventualité, un examen attentif des équations définissant les $A_{n}, B_{n}, \ldots$ etc. montre que les coefficients des inconnues décroissent d'autant plus vite avec $1 / n$ que la profondeur relative devient plus grande. Il s'ensuit que l'approximation que nous avons adoptée:

$$
\sum_{1}^{\infty} \sim{\frac{V^{N}}{N}}^{N}
$$

sera d'autant meilleure que la longueur d'onde L, correspondant à la profondeur $h$ fixée, est plus petite. Or, cela exige que la période $\mathrm{T}$ soit petite. On interprète ainsi a priori un fait déjà observé; à savoir que la précision de notre caleul approché augmente lorsque, toutes choses égales d'ailleurs, $\mathrm{T}$ diminue.

Il reste maintenant à apprécier l'influence à cet égard du paramètre $l$. A ce sujet, nous ne pouvons avancer que des hypothèses. Nous croyons que notre approximation doit devenir meilleure lorsque $l$ augmente. Cette propriété tiendrait à ce que la régularité du mouvement du liquide dans $D_{0}$ augmente lorsque $l$ croît. D'une manière plus précise, l'influence des termes perturbateurs existant pour $x=0$ sur le régime dans le voisinage de $x=l$ diminue avec $1 / l$ et devient insensible pour $l>>\Lambda$ ( $\Lambda$ étant la longueur d'onde de la houle de période $T$ en profondeur uniforme $\left(h-h_{0}\right)$. On constale alor's que plusieurs coefficients du système (4) devien- 
nent très petits, surtout pour $n \geqq 2$, ce qui justifierait notre hypothèse. Or, dans les expériences de Jolas, $l \sim \Lambda$; la condition $l>>\Lambda$ n'était donc pas réalisée.

En résumé, notre méthode de calcul approchẻ serait d'autant plus précise, pour $h$ et $h_{0}$ fixés, que la période $T$ serait plus petite et la longueur $l$ plus grande.

Passons maintenant aux théories globales. Rappelons qu'elles reposent toutes sur des hypothèses a priori concernant la répartition verticale des vitesses. Or, ces approximations sont d'a'utant meilleures que la longueur d'onde est plus grande; autrement dit, le domaine de validité de ces théories serait celui des houles dites «longues », c'est-à-dire des ondes à grande période. Nos résultats numériques semblent en accord avec cette interprétation.

Il reste maintenant à discuter de la validité physique de la théorie linéaire. Dans la première partie, nous avons longuement insisté sur la signification analytique de cette validité : à savoir, la rapidité de la convergence des séries de Poincaré, représentant la solution et procédant suivant les puissances positives de la cambrure $a_{1} /$ L. Rappelons qu'admettre cette convergence, c'est déjà postuler que ce paramètre est petit; mais pour que les fonctions inconnues soient bien représentées par leurs termes linéaires seuls, il faut supposer $a_{1} / \mathrm{L}$ plus faible encore. La validité physique de la théorie linéaire revient donc à l'étude de l'influence de la cambrure sur la nature des approximations. Nous avons déjà indiqué les raisons qui nous incitaient à penser que, pour assurer la validité de la théorie linéaire dans notre cas, ce paramètre doit être d'autant plus petit que la profondeur relative est plus petite; répétons que si $h / L$ est petit, les termes d'ordre supérieur au premier peuvent devenir considérables, même si $a_{1} / \mathrm{L}$ est petit. Pour illustrer ce fait d'une manière frappante, nous renverrons le lecteur à un article de Chabert d'Hières [Cf. (8)], relatif au clapotis parfait en profondeur uniforme. Nous supposerons que ces propriétés qualitatives demeurent encore valables dans notre cas. Voici alors une difficulté qui se présente effectivement dans l'étude des effets de passage des ondes sur un seuil. Supposons que, pour une période $\mathbf{T}$ donnée, la profondeur relative dans $D_{1,2}$ et $D_{3}$ soit assez grande et la cambrure assez faible pour que, dans ces domaines, la théorie linéaire représente correctement les phénomènes. Mais il peut se faire que la profondeur relative $\left(h-h_{0}\right) / \Lambda$ dans $\mathrm{D}_{0}$ soit faible; c'est ce qui se produit dans le cas des seuils élevés. Alors, dans $\mathrm{D}_{0}$, les termes non linéaires deviendront importants. Car, dans le cas actuel, et contrairement à ce qui se produit avec le schéma de Macagno, - une portion de la frontière de l'image de $D_{0}$ dans le plan du mouve- ment est constituce par la surface libre. Or, c’est précisément la condition frontic̀re le long de celle-ci qui assure la croissance rapide des termes non linéaires avec $\Lambda /\left(h-h_{0}\right)$. La théorie est alors à reprendre; car, le potentiel $\Phi_{0}$ dans $D_{0}$ ne peut plus être représenté avec une approximation suffisante par son développement (2).

J. Kravtchenko - à qui nous devons ces indications d'ordre qualitatif - est parti de ces constatations pour ébaucher la théorie d'un phénomène dont l'intérêt lui a été signalé par J.J. Stoker et dont voici la description sommaire. Les océanographes ont observé les effets de passage d'une houle incidente de période 'T donnée sur un banc de sable. On constate alors l'apparition à l'aval d'un train d'ondes de période $\mathrm{T} / n$, qui se superposent à la houle transmise de période $T$. Il est clair que la théorie linéaire est impuissante à interpréter un phénomène de cette nature. Voici un schéma non linéaire relatif à la configuration que nous étudions, qui constitue, peut-être, le point de départ de l'explication des faits précédents. On a vu que si $\left(h-h_{0}\right) / \Lambda$ est assez petit, on verra apparaître dans le domaine $D_{0}$ des oscillations harmoniques non linéaires de période $T / n$; pour satisfaire les conditions aux limites le long des segments $x=0$ et $x=l$, qui assurent la continuité du potentiel dans $D=D_{1,2}+D_{0}+D_{3}$, on devra introduire dans $D_{3}$ et $D_{1,2}$ à côté de $\Phi_{3}$ et $\Phi_{1}+\Phi_{2}$, des potentiels harmoniques additifs du type :

$$
\text { T }(x, z) e^{i n k t}
$$

On montre aisément que si la profondeur relative $h / L$ est assez grande, ces potentiels donnent naissance à des houles harmoniques linéaires de période $T / n$; de plus, celles-ci ne peuvent être sensibles que dans $\mathrm{D}_{3}$. Nous avons aussi une explication possible du phénomène signalé par Stoker, qui consisterait essentiellement en ce qu'une oscillation linéaire de période $T$ à l'amont de $\mathrm{S}$ donnerait naissance à l'aval à un train d'ondes linéaires de périodes $\mathrm{T} / n$ par un mécanisme non linéaire, localisé dans la masse liquide au-dessus du seuil. A la demande de J. Kravtchenko, nous avons tenté de construire une théorie de ce phénomène; mais la longueur des calculs nous a contraint à abandonner cette tentative. Toutefois, nous avons pu obtenir quelques résultats explicites dans le cas particulier [Cf. (9)] d'un seuil semi-indéfini, autrement dit correspondant à la valeur $l=\infty$ de notre paramètre actuel. Il semble que les prévisions de Kravtchenko soient confirmées; mais de nouvelles et pénibles recherches sont encore nécessaires pour conclure avec certitude. De plus, les expériences de Jolas sont en accord qualitatif avec ces vues. Voici quelques nombres extraits 
de (2), correspondant à la géométrie de $\mathrm{S}$ étudiée ci-dessus. Pour $\mathrm{T}=0,816 \mathrm{~s}$, on n'a observé d'harmoniques sensibles ni à l'aval ni à l'amont de $S$. Pour $T=1,104 \mathrm{~s}$, on n'a pas relevé d'harmoniques appréciables à l'amont de $S$; par contre, à l'aval, ont apparu des harmoniques $\mathrm{T} / 2$ et $\mathrm{T} / 3$ d'amplitude de l'ordre $1,4 a_{1}$; l'harmonique $\mathrm{T} / 10$ avait encore une amplitude sensible. Dans tous ces essais, $a_{1}$ était de l'ordre du centimètre.
Ainsi la nature du phénomène à l'aval de $\mathrm{S}$ scra des plus complexes, d'autant que les harmoniques linéaires engendrent des ondes d'interaction non linéaires dont on trouvera la théorie dans la thèse de A. Daubert (Cf. [10]).

Les effets de passage d'une houle sur un seuil donneront sans doute lieu à beaucoup d'études avant d'être connus avec précision; notre travail ne constitue à cet égard qu'une première étape.

\section{BIBLIOGRAPHIE}

[1] K. Takano. Effet de passage d'une houle linéaire plane sur un seuil. C.R.A.S., t. 248, 1959, pp. 17681771.

[2] P. Jolas. Passage de la houle sur un seuil. La Houille Blanche, $\mathrm{n}^{\circ} 2,1960$, pp. 148-152.

[3] H. Jefrereys. Wave Report, $\mathrm{n}^{\circ}$ 3. Minist. of Supply, Great Britain, 1944.

[4] Le MÉhauté. Two dimensional seiches in a basin subjected to incident waves. Proc. V'th Conf. Coast, Eng., Grenoble, 1954, pp. $119-150$.

[5] J. Valembors. Proc. Minnesota Intern. Hydraul. Conv., Minnesota, 1953, pp. 193-199.

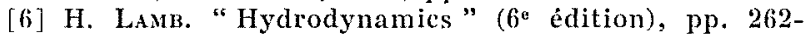
263, Dover Publications.
[7] W. R. DEAn. On the reflexion of surface waves by a submerged plane barrier.

Proc. Camb. Phil. Soc., 1945, 41, pp. 231-238.

[8] Chabert D'Hières. Etude du clapotis. Communication à la S.H.F., juin 1959 .

La Houille Blanche, $\mathrm{n}^{\circ}$ 2, 1960, pp. 153-163.

[9] K. Takano. Effets de second ordre d'un seuil semiindéfini sur une houle irrotationnelle. C.R.A.S., t. 249, 1959, pp. 622-624.

[10] A. Daubert. Thèse, Université de Grenoble. Théorie approchée de la houle pure et de la houle complexe. (A paraître dans les Publ. Sci. et Tech. du Minist. de l'Air.)

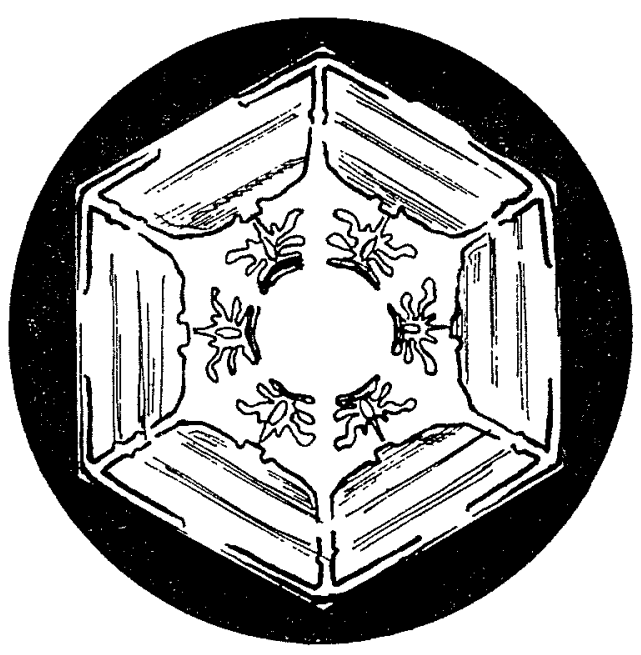

